\title{
PERIODIC OPTICAL VARIABILITY OF RADIO-DETECTED ULTRACOOL DWARFS
}

\author{
L. K. Harding ${ }^{1,2,6}$, G. Hallinan ${ }^{2}$, R. P. Boyle ${ }^{3}$, A. Golden ${ }^{1,4}$, Navtej Singh ${ }^{1}$, \\ B. SheEhan ${ }^{1}$, R. T. Zavala ${ }^{5}$, AND R. F. Butler ${ }^{1}$ \\ ${ }^{1}$ Centre for Astronomy, National University of Ireland, Galway, University Road, Galway, Ireland; 1kh@astro.caltech.edu \\ ${ }^{2}$ Cahill Center for Astrophysics, California Institute of Technology, 1200 East California Boulevard, MC 249-17, Pasadena, CA 91125, USA \\ ${ }^{3}$ Vatican Observatory Research Group, Steward Observatory, University of Arizona, Tucson, AZ 85721, USA \\ ${ }^{4}$ Department of Genetics (Computational Genetics), Albert Einstein College of Medicine, Bronx, NY 10461, USA \\ ${ }^{5}$ United States Naval Observatory, Flagstaff Station, Flagstaff, AZ 86001, USA \\ Received 2012 June 23; accepted 2013 September 27; published 2013 November 27
}

\begin{abstract}
A fraction of very low mass stars and brown dwarfs are known to be radio active, in some cases producing periodic pulses. Extensive studies of two such objects have also revealed optical periodic variability, and the nature of this variability remains unclear. Here, we report on multi-epoch optical photometric monitoring of six radio-detected dwarfs, spanning the $\sim$ M8-L3.5 spectral range, conducted to investigate the ubiquity of periodic optical variability in radio-detected ultracool dwarfs. This survey is the most sensitive ground-based study carried out to date in search of periodic optical variability from late-type dwarfs, where we obtained $250 \mathrm{hr}$ of monitoring, delivering photometric precision as low as $\sim 0.15 \%$. Five of the six targets exhibit clear periodicity, in all cases likely associated with the rotation period of the dwarf, with a marginal detection found for the sixth. Our data points to a likely association between radio and optical periodic variability in late-M/early-L dwarfs, although the underlying physical cause of this correlation remains unclear. In one case, we have multiple epochs of monitoring of the archetype of pulsing radio dwarfs, the M9 TVLM 513-46546, spanning a period of $5 \mathrm{yr}$, which is sufficiently stable in phase to allow us to establish a period of $1.95958 \pm 0.00005 \mathrm{hr}$. This phase stability may be associated with a large-scale stable magnetic field, further strengthening the correlation between radio activity and periodic optical variability. Finally, we find a tentative spin-orbit alignment of one component of the very low mass binary, LP 349-25.
\end{abstract}

Key words: binaries: general - brown dwarfs - instrumentation: photometers - stars: low-mass - stars: magnetic field - stars: rotation

Online-only material: color figures

\section{INTRODUCTION}

Beyond spectral type $\gtrsim \mathrm{M} 7$ (ultracool dwarfs; Kirkpatrick et al. 1997), $\mathrm{H} \alpha$ and X-ray luminosities drop sharply, signaling that chromospheric and coronal heating becomes less efficient, even in the presence of rapid rotation (Mohanty \& Basri 2003; West et al. 2004; Reiners \& Basri 2008; West \& Basri 2009). Despite this reduction in quiescent emission, a number of $\mathrm{H} \alpha$ and X-ray flares have been detected, indicating that chromospheric and coronal activity is indeed present (Reid et al. 1999; Gizis et al. 2000; Rutledge et al. 2000; Liebert et al. 2003; Fuhrmeister \& Schmitt 2004; Rockenfeller et al. 2006a). Surprisingly, given the absence of quiescent emission at higher energies, Berger et al. (2001) reported persistent radio emission from LP 944-20 (M9)-the first detection of radio emission from a brown dwarf, orders of magnitude higher than the expected flux (Güdel \& Benz 1993).

To date, quiescent radio emission has been detected from 10 ultracool dwarfs (Berger et al. 2001, 2005, 2009; Berger 2002, 2006; Burgasser \& Putman 2005; Osten et al. 2006; Phan-Bao et al. 2007; Hallinan et al. 2006, 2007; Antonova et al. 2007; Route \& Wolszczan 2012). Probably the most surprising aspect of this radio activity has been the detection of periodic $100 \%$ circularly polarized pulses (Hallinan et al. 2007, 2008; Berger et al. 2009). Observations by Hallinan et al. (2007) of TVLM 513-46546 (henceforth TVLM 513), reveal electron cyclotron maser (ECM) emission as the mechanism responsible for these $100 \%$ circularly polarized periodic pulses,

\footnotetext{
6 Now at Caltech.
}

implying kilogauss $(\mathrm{kG})$ magnetic field strengths in a largescale stable magnetic field configuration. This is consistent with the confirmation of $\mathrm{kG}$ magnetic field strengths for ultracool dwarfs via Zeeman broadening observations (Reiners \& Basri 2007). Although these observations confirmed the ECM process to be the cause of the polarized periodic emission, it is still unclear as to which mechanism (incoherent or coherent) is driving the quiescent component of the radio emission, and incoherent gyrosynchrotron emission has alternatively been invoked (Berger 2006; Osten et al. 2006).

Ultracool dwarfs have also exhibited periodic variability in the optical regime. These investigations have yielded both optical and infrared variability, where modulation at the expected rotation period has been found in various studies (Clarke 2002b; Koen 2006; Rockenfeller et al. 2006a; Lane et al. 2007; Littlefair et al. 2008). Aperiodic variability, as well as periodic modulations on timescales not associated with rotation, have been inferred (Gelino et al. 2002; Lane et al. 2007; Maiti 2007). Typically, this variability has been attributed to magnetic spots on the surface of the dwarf, or the presence of atmospheric dust, or both. For higher temperature ultracool dwarfs (specifically late-M and early-L dwarfs), the presence of magnetic spots and other magnetic related activity, as seen for earlier M-dwarfs, may be present (Rockenfeller et al. 2006a; Lane et al. 2007). Littlefair et al. (2008) reported sinusoidal variability of the M9 dwarf TVLM 513, with a period of $\sim 2 \mathrm{hr}-$ a period consistent with the radio pulsing and optical periodicity previously obtained by Hallinan et al. (2006) and Lane et al. (2007), respectively. However, their light curves (Sloan $g^{\prime}$ and Sloan $i^{\prime}$ ) were anticorrelated, which seemed to refute the proposed model 
Table 1

Summary of Campaign Sample Properties

\begin{tabular}{|c|c|c|c|c|c|c|c|c|c|}
\hline Source & SpT & $\begin{array}{l}\text { Distance } \\
\text { (pc) } \\
\text { (3) }\end{array}$ & $I(\mathrm{mag})$ & $\begin{array}{c}\log \\
\left(L_{\mathrm{bol}} / L_{\odot}\right) \\
(5)\end{array}$ & $\begin{array}{c}v \sin i \\
\left(\mathrm{~km} \mathrm{~s}^{-1}\right) \\
(6)\end{array}$ & Lithium? & $\begin{array}{l}\text { Est. Mass } \\
\qquad M_{\mathrm{tot}} \\
\left(M_{\odot}\right) \\
(8)\end{array}$ & References & $\begin{array}{l}\text { Radio } \\
\text { Disc. } \\
\text { Ref. } \\
(10)\end{array}$ \\
\hline LP $349-25 \mathrm{AB}$ & $\sim \mathrm{M} 8+\mathrm{M} 9^{\mathrm{a}}$ & $13.10 \pm 0.28$ & 12.40 & $-3.19 ;-3.34$ & $55 \pm 2 ; 83 \pm 3$ & No & $0.121 \pm 0.009$ & $1-3$ & 21 \\
\hline 2M J0746AB & L0+L1.5 & $12.20 \pm 0.05$ & 15.03 & $-3.64 ;-3.77$ & $19 \pm 2 ; 33 \pm 2$ & No & $0.151 \pm 0.003$ & $2,4-6$ & 22 \\
\hline LSR J1835 & M8.5 & $\sim 6.0$ & 12.90 & -3.51 & $50 \pm 5$ & $?$ & $<0.083 ?$ & $7-9$ & 23 \\
\hline TVLM 513 & M9 & $\sim 10.5$ & 15.10 & -3.65 & $\sim 60$ & No & $>0.06$ & $4,10-13$ & 24 \\
\hline BRI 0021 & M9.5 & $\sim 11.5$ & 15.02 & -3.40 & $\sim 34$ & No & $<0.06$ & $11,14-17$ & 24 \\
\hline 2M J0036 & L3.5 & $\sim 8.8$ & 16.05 & -3.98 & $\sim 37$ & No & $0.06-0.074$ & $4,5,18-20$ & 25 \\
\hline
\end{tabular}

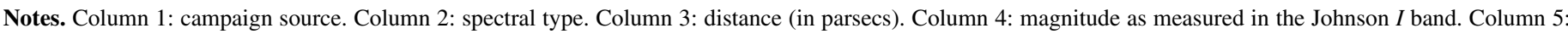

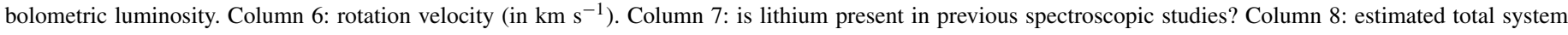

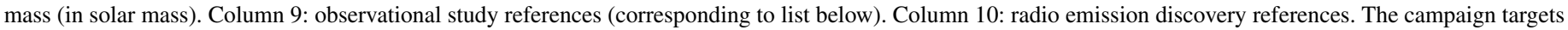

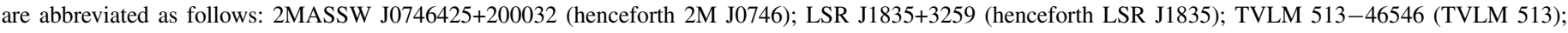
BRI 0021-0214 (henceforth BRI 0021) and 2MASS J00361617+1821104 (henceforth 2M J0036).

${ }^{a}$ LP 349-25 may be either M7.5+M8.5 or M8+M9 as outlined by Forveille et al. (2005).

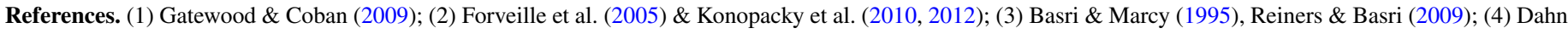

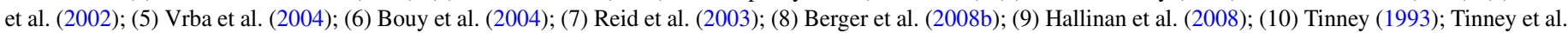

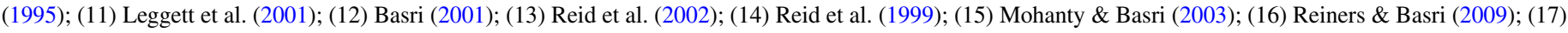

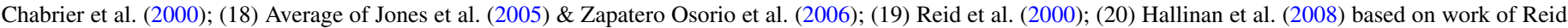
et al. (2000) and Vrba et al. (2004); (21) Phan-Bao et al. (2007); (22) Antonova et al. (2008); (23) Berger (2006); (24) Berger (2002); (25) Berger et al. (2005).

of starspots at that time as the cause for the optical variability. Instead, they argued that this anticorrelated signal was likely due to photospheric dust coupled with stellar rotation. Indeed, magnetic activity, as signaled by $\mathrm{H} \alpha$, decreases further after the M/L transition (West et al. 2004); therefore, in most cases, optical variability has been attributed to the expected presence of dust in the dwarf's atmosphere (Bailer-Jones \& Mundt 2001; Martín et al. 2001; Gelino et al. 2002; Enoch et al. 2003; Maiti 2007; Littlefair et al. 2008; Goldman et al. 2008; Clarke et al. 2008).

It is notable that two of the ultracool dwarfs found to be periodically variable in the optical (Lane et al. 2007) are also known to be members of the small sample known to be pulsing radio sources (Hallinan et al. 2007, 2008). Motivated by this, we have commenced a campaign to investigate whether optical periodic variability is a signature property of radiodetected ultracool dwarfs. To this end, we employed the customdeveloped Galway Ultra Fast Imager (GUFI) mk.II photometer, as well as the VATT 4K CCD Imager on the $1.83 \mathrm{~m}$ Vatican Advanced Technology Telescope (VATT), ${ }^{7}$ to photometrically monitor all of the radio emitting ultracool dwarfs observable from the VATT site. Throughout the campaign, data were also obtained from the $1.0 \mathrm{~m}$ and $1.55 \mathrm{~m}$ telescopes at the USNO, ${ }^{8}$ as well as the $1.52 \mathrm{~m}$ telescope at the Loiano Observatory in Bologna, Italy.

\section{SAMPLE}

In the following sections we discuss each target with respect to any previous radio and optical emission. A list of the campaign sample, as well as a summary of individual target information, is shown in Table 1. These are categorized in order of ascending spectral type; we also outline details of the respective observation campaigns. Our target sample consists

\footnotetext{
7 The Vatican Advanced Technology Telescope (VATT) telescope facility is operated by the Vatican Observatory, and is part of the Mount Graham International Observatory.

8 Information regarding the United States Naval Observatory (USNO) telescopes can be found here: http://www.usno.navy.mil/USNO.
}

of those dwarfs which have been previously detected as radio sources and are visible from the VATT observatory site. The general capabilities of each detector used for the campaign are outlined in Section 3. Dwarfs which have been detected as optically variable sources in other work were also included for verification, and to assess the stability of these optical signals over time scales of years.

\subsection{Binary Systems}

We selected two very low mass (VLM) binary stars at the M/L transition for our campaign-LP 349-25 and 2M J0746. These objects were of particular interest, since they are the only binary dwarfs reported thus far to exhibit radio emission in the VLM binary regime (Phan-Bao et al. 2007; Antonova et al. 2008; Osten et al. 2009; Berger et al. 2009), defined to be $M_{\text {tot }} \leqslant 0.185 M_{\odot}$ (Close et al. 2003). Furthermore, both objects were subject to high-precision dynamical mass measurements (Dupuy et al. 2010; Konopacky et al. 2010), and more recently, a large campaign was carried out to establish the individual rotational velocities of each binary component (Konopacky et al. 2012) — the first resolved Adaptive Optics (AO) measurements of this kind. Based on these dynamical mass and rotational velocity measurements, an accurate period of rotation provides the means of assessing the system's orbital coplanarity. Moreover, a range of radii can also be estimated. Importantly, Harding et al. (2013) have recently reported alignment of the spin-orbital axes of $2 \mathrm{M} \mathrm{J} 0746 \mathrm{AB}$. Thus, the discovery of a rotation period from the binary LP 349-25 has allowed us to investigate this possible alignment for another VLM system.

\subsection{1. $L P 349-25 A B(M 8 V+M 9 V)$}

The M tight binary dwarf LP $349-25$ was reported as a quiescent radio source by Phan-Bao et al. (2007) and Osten et al. (2009); however, no radio pulsing has been found thus far. More recently, a rotational velocity study carried out by Konopacky et al. (2012) of individual components of VLM binaries, including LP 349-25, yielded a $v \sin i$ of $55 \pm$ $2 \mathrm{~km} \mathrm{~s}^{-1}$ and $83 \pm 3 \mathrm{~km} \mathrm{~s}^{-1}$ for LP $349-25 \mathrm{~A}$ and LP 
349-25B, respectively. Under the assumption of a rotational axis which is orthogonal to the orbital plane (Hale 1994), the inferred equatorial velocities are $\sim 62 \mathrm{~km} \mathrm{~s}^{-1}$ and $\sim 95 \mathrm{~km} \mathrm{~s}^{-1}$, respectively. This would make LP $349-25 \mathrm{~B}$ the fastest rotating low mass object yet discovered.

Thus far, no optical variability has been detected for LP 349-25. Therefore, we chose to monitor the binary to investigate the presence of optical variability. We used VATT $R$-band and $I$-band broadband filters for observations with GUFI mk.II over the course of three separate epochs, for a total of $\sim 64 \mathrm{hr}$, spanning $\sim 1.2 \mathrm{yr}$.

\subsection{2. $2 M A S S W J 0746425+200032 A B(L 0+L 1.5)$}

2M J0746 is an L dwarf binary with a separation of $\sim 2.7 \mathrm{AU}$ (Reid et al. 2001). The first detection of confirmed radio emission was reported by Antonova et al. (2008) during a $2 \mathrm{hr}$ observation. Following this observation, Berger et al. (2009) reported periodic radio emission of $2.07 \pm 0.002 \mathrm{hr}$, as well as quasi-sinusoidal periodic $\mathrm{H} \alpha$ emission with the same period. Berger et al. (2009) proposed that the source of the periodicity in both cases was coming from the same component of the binary,

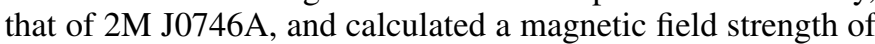
$\sim 1.7 \mathrm{kG}$, which was in agreement with Antonova et al. (2008).

Recently, Konopacky et al. (2012) reported the first resolved $v \sin i$ measurements of the system. They measure a $v \sin$ $i$ of $19 \pm 2 \mathrm{~km} \mathrm{~s}^{-1}$, and $33 \pm 2 \mathrm{~km} \mathrm{~s}^{-1}$, for $2 \mathrm{M} \mathrm{J0746A}$ and $2 \mathrm{M} \mathrm{J} 0746 \mathrm{~B}$, respectively. Previously, in terms of rotation period measurement in optical photometry, there were only rough estimates based on unresolved $v \sin i$ data (with reported periods of 1.84-5.28 hr; see Bailer-Jones 2004), as well as some photometric variability which was detected by Clarke et al. (2002a), showing weak evidence of periodicity of a few hours. Harding et al. (2013) reported a period of $3.32 \pm 0.15 \mathrm{hr}$ for $2 \mathrm{M}$ J0746A, inferring that Berger et al. (2009) in fact detected the secondary in the radio. This refuted the claimed radius of $0.78 \pm$ $0.1 R_{J}$ for $2 \mathrm{M} \mathrm{J} 0746 \mathrm{~A}$, which Harding et al. (2013) demonstrate to be $0.99 \pm 0.03 R_{J}$.

A total of $\sim 62 \mathrm{hr}$ of multiple epoch $I$-band observations were obtained over $\sim 2$ yr to investigate the long-term behavior of the optical variability on timescales of years. These observations were taken with the VATT 4K system as well as the GUFI mk.II photometer.

\subsection{Single Systems}

\subsubsection{LSR J1835+3259 (M8.5)}

The ultracool dwarf, LSR $\mathrm{J} 1835$, is a rapid rotator with a $v$ $\sin i$ of $50 \pm 5$ (Berger et al. 2008b). Berger (2006) detected radio emission from LSR J1835 during a $\sim 2 \mathrm{hr}$ observation and proposed incoherent gyrosynchrotron radiation was responsible with an associated field strength of $<30 \mathrm{G}$. Hallinan et al. (2008) later observed the dwarf for $11 \mathrm{hr}$ using the VLA, and reported persistent $100 \%$ circularly polarized coherent pulses of radio emission with a period of $2.84 \pm 0.01 \mathrm{hr}$, which they attributed to the dwarf's rotation period. They argue in favor of ECM emission as the dominant source of the pulsed radio emission, requiring magnetic fields of $\sim 3 \mathrm{kG}$.

Based on the above radio activity of LSR J1835, we decided to further investigate the presence of such variability at optical wavelengths, and whether it was periodic in nature like the optical periodic variability presented by Lane et al. (2007) for the M9 dwarf TVLM 513. We conducted observations over a period of $\sim 3 \mathrm{yr}$, encompassing three separate epochs. Initial epochs were taken as test data only for the GUFI mk.I system in 2006 July in the Johnson $I$ band, using the $1.52 \mathrm{~m}$ telescope in Loiano, Bologna, Italy. We also include Johnson $I$-band and $R$-band data from the USNO $1.55 \mathrm{~m}$ telescope in Flagstaff, Arizona, obtained by group members in 2006 September. Finally, we observed the dwarf in the VATT $I$ band with the GUFI mk.II system on the 1.83 m VATT telescope, Mt. Graham, Arizona, to confirm its periodic nature in 2009 June. The three epochs contain $\sim 33 \mathrm{hr}$ of observations on source.

\subsubsection{TVLM 513-46546 (M9)}

TVLM 513 is one of the most rapidly rotating ultracool dwarfs discovered thus far with a rotation rate of $\sim 60 \mathrm{~km} \mathrm{~s}^{-1}$ (Basri 2001). All the same, only weak levels of $\mathrm{H} \alpha$ have been found in its spectrum (Martín et al. 1994; Reid et al. 2001; Mohanty \& Basri 2003), with no X-ray detections reported so far.

Berger (2002) and Osten et al. (2006) detected transient radio emission from TVLM 513, however, no obvious flaring was found. Hallinan et al. (2006) then reported persistent periodic radio emission with a period of $\sim 2 \mathrm{hr}$. Following this, Hallinan et al. (2007) revealed periodic bursts of radio emission with a period of $\sim 1.96 \mathrm{hr}$-confirming the presence of $\mathrm{kG}$ magnetic field strengths based on broadband, ECM coherent radio emission. These observations were conducted simultaneously to a photometric monitoring campaign by Lane et al. (2007), who also detected a periodic signal of $\sim 1.96 \mathrm{hr}$ in photometric I-band data (attributed to magnetic spots), establishing that the periodicity was due to the rotational modulation of the star, as put forward by Hallinan et al. (2006). However, Littlefair et al. (2008) instead propose that atmospheric dust was responsible, after reporting anti-correlated Sloan $g^{\prime}$ and $i^{\prime}$ periodic variability of the M9 dwarf. Periodic $\mathrm{H} \alpha$ and $\mathrm{H} \beta$ variability has also been reported (Berger et al. 2008a), perhaps indicating the presence of localized heating in the dwarf's chromospheric regions.

We observed TVLM 513 in optical photometric VATT I-band observations with GUFI mk.II on VATT in 2009 June, in addition to three additional $I$-band epochs in 2011 February and April, and in 2011 May using the VATT 4K CCD and a Sloan $i^{\prime}$ filter. Data taken by members of the group using the USNO $1.0 \mathrm{~m}$ telescope is also included, from an epoch in 2008, and earlier VATT data obtained in 2006. Therefore, this baseline extends for $\sim 5 \mathrm{yr}$ encompassing $\sim 53 \mathrm{hr}$ of data.

\subsubsection{BRI $0021-0214(M 9.5)$}

In a campaign investigating magnetic activity in ultracool dwarfs, Berger et al. (2010) found steady and variable $\mathrm{H} \alpha$ emission from BRI 0021 on a $\sim 0.5-2 \mathrm{hr}$ timescale, albeit no detected radio emission, despite previous low-level detections of radio emission (Berger 2002). Reid et al. (1999) also reported a weak $\mathrm{H} \alpha$ flare. Other optical variability has been reported by Martín et al. (2001), who find I-band variability during multi-epoch photometric observations with some evidence of periodicity ( $\sim 20 \mathrm{hr}$ and $\sim 4.8 \mathrm{hr}$ ) in their analysis. They argue that since the dwarf appeared to have low levels of magnetic activity, the variability was probably not due to surface spots, but rather due to dust clouds in the dwarf's atmosphere-since the presence of silicate and iron clouds are expected based on the dwarf's spectrum (Chabrier et al. 2000). It is a rapidly rotating dwarf with a $v \sin i \approx 34 \mathrm{~km} \mathrm{~s}^{-1}$ (Reid et al. 1999; Mohanty \& Basri 2003).

Based on the above radio and optical studies, we observed the dwarf in broadband optical photometry with GUFI mk.II, and 
obtained $\sim 28 \mathrm{hr}$ of $I$-band data over three epochs of $\sim 1.2 \mathrm{yr}$ of separation.

\subsubsection{MASS J00361617+1821104 (L3.5)}

$2 \mathrm{M} \mathrm{J0036} \mathrm{is} \mathrm{a} \mathrm{radio} \mathrm{active} \mathrm{ultracool} \mathrm{dwarf} \mathrm{with} \mathrm{rotation}$ velocity estimates of $\sim 15 \mathrm{~km} \mathrm{~s}^{-1}, 38 \mathrm{~km} \mathrm{~s}^{-1}$, and $36 \mathrm{~km} \mathrm{~s}^{-1}$ based on the studies of Schweitzer et al. (2001), Jones et al. (2005), and Zapatero Osorio et al. (2006), respectively.

Berger et al. (2005) confirmed the presence of highly variable, periodic radio emission, with a period of $\sim 3 \mathrm{hr}$. This level of radio emission violated the Güdel-Benz relationship by many orders of magnitude (see Güdel \& Benz 1993, Benz \& Güdel 1994). They interpret the emission as incoherent gyrosynchrotron radiation, with a corresponding magnetic field strength of $175 \mathrm{G}$. However, Hallinan et al. (2008) reported 2M J0036 to once again be a persistent source of radio emission, and based on the periodic presence of $100 \%$ circularly polarized emission, ruled out gyrosynchrotron radiation and confirmed ECM emission as the mechanism responsible for the pulsed radio emission. This required a magnetic field strength of at least $1.7 \mathrm{kG}$, which was the first confirmation of $\mathrm{kG}$ magnetic field strengths for an L dwarf.

Prior to these observations, Lane et al. (2007) conducted photometric I-band observations of $2 \mathrm{M} \mathrm{J0036}$ and found the dwarf to be photometrically variable, with a periodicity of $\sim 3 \mathrm{hr}$, arguing that magnetic spots on the surface of the dwarf, coupled with the rotation of the star, were a likely source of the periodicity. Some evidence of aperiodic variability was also present, which they attribute to dust clouds in the cooler L dwarf atmosphere.

We chose to observe 2M J0036 in optical photometry in the same optical band as Lane et al. (2007) to determine whether the optical periodicity was present over timescales of years. We used GUFI mk.II on VATT at $I$-band wavelengths, for two nights in 2010 December, for a total of $\sim 10 \mathrm{hr}$.

\section{OPTICAL OBSERVATIONS}

\subsection{GUFI mk.II: The Galway Ultra Fast Imager Photometer}

The GUFI instrument was originally commissioned by astronomers in NUI Galway as an optical photometer capable of high-time resolution imaging (Sheehan \& Butler 2008). We modified the GUFI mk.II system (hereafter GUFI) to be compatible with the $1.83 \mathrm{~m}$ VATT on Mt. Graham, Arizona, where it is currently stationed as a visitor instrument. The system uses the Andor iXon DV887 EM-CCD camera, which has a CCD97 thinned back-illuminated sensor from e2v technologies, hosting $>90 \%$ quantum efficiency (QE) with a native $512 \times 512$ frame transfer sensor. It offers variable readout rates up to $10 \mathrm{MHz}$ and can operate full-frame at 34 frames s $^{-1}$ (fps) and up to $526 \mathrm{fps}$ in a windowed configuration. The native field of view (FOV) of GUFI at the VATT Gregorian focus is $\sim 1.7 \times 1.7$ with a corresponding plate scale of 0.2 pixel $^{-1}$. Focal reducer options for wider fields are limited by the short VATT back focal distance of $50.8 \mathrm{~mm}$, but GUFI provides near-infrared (NIR) and visible-optimized focal reducers, offering a FOV of $\sim 3^{\prime} \times 3^{\prime}$ and a larger plate scale of 0.35 pixel $^{-1}$. The VATT telescope offers the full range of Johnson and Sloan filter sets, as well as Vilnius interference filters. Thus, GUFI had an effective spectral sensitivity during this campaign of 3000-10,000 $\AA$ (based on the QE). The great advantages of GUFI for this study are its $100 \%$ observing duty cycle (with a $\sim 2$ ms readout rate), very low readout noise, and high $\mathrm{QE}$.

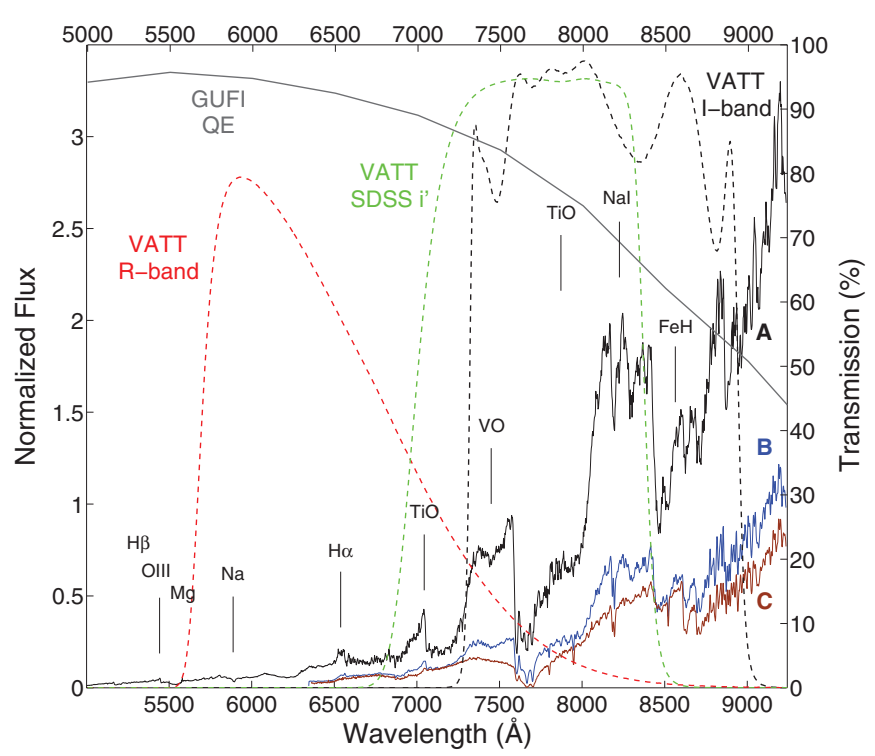

Figure 1. Filter transmission curves with overplotted spectra of an M8 dwarf (A), an L0.5 dwarf (B), and an L3.5 dwarf (C) - the spectral range which encompasses our study. The spectra have been normalized ( $y$-axis, left) by the peak flux of the M8 dwarf spectra at $9200 \AA$. The optical filters used in this study are shown by the dashed lines of wavelength ( $x$-axes) vs. $\%$ transmission ( $y$-axis, right): VATT $R$ band ( $\sim 5600-8800 \AA$; red), Sloan $i^{\prime}(\sim 6500-9500 \AA$; green), and VATT $I$ band ( 7200-9100 $\AA$; black). We also include the GUFI photometer's QE curve (solid gray line) to highlight transmission for the different wavebands.

(A color version of this figure is available in the online journal.)

\subsection{The VATT 4 K Imager}

The VATT 4K CCD camera is the primary in-house photometer stationed at VATT. It houses a back-illuminated STA0500A CCD with a transfer sensor of $4064 \times 4064$ pixels, a native FOV of $\sim 12$ '.5 $\times 12$ '.5, and a plate scale of $0.188 \mathrm{pixel}^{-1}$. The standard readout rate for the camera is $50 \mathrm{~s}$; however, faster readout rates can be achieved based on the level of windowing, and binning options, applied to the frame.

\subsection{The USNO Detectors}

Some observations, as outlined in the relevant target details in Section 2, were obtained with the USNO $1.0 \mathrm{~m}$ and USNO $1.55 \mathrm{~m}$ telescopes. The new $2 \mathrm{k}$ camera on the $1.0 \mathrm{~m}$ telescope has a FOV of $23 ! 2 \times 23 ! 2$ and a pixel scale of 0.68 pixel $^{-1}$. We used the Tek $2 \mathrm{k}$ camera on the $1.55 \mathrm{~m}$, which has a corresponding FOV of $11^{\prime} .3 \times 11^{\prime} .3$ with a pixel scale of 0 .'33 pixel $^{-1}$.

\subsection{Observations and Data Reduction}

The observation campaigns were carried out between 2006 May and 2011 May. We used the VATT R-Harris ( 5600-8800 $\AA)$ filter, the Sloan $i^{\prime}(\sim 6500-9500 \AA)$ filter, the Johnson I-band filter $(\sim 7000-11,000 \AA)$, and the VATT I-Arizona ( 7200-9100 A) filter-for selected targets (Table 2). Transmission curves for each filter are shown in Figure 1. The campaign encompassed observations to search for periodic variability of all radio-detected dwarfs listed in Table 2, that were visible from the VATT observatory site $\left(32^{\circ} 42^{\prime} 4^{\prime \prime} .78 \mathrm{~N}\right.$ $\left.109^{\circ} 53^{\prime} 32^{\prime \prime} .5 \mathrm{~W}\right)$. We also obtained data from the $1.52 \mathrm{~m}$ telescope, in Loiano, Bologna, Italy, as well as the $1.0 \mathrm{~m}$ and $1.55 \mathrm{~m}$ USNO telescopes, in Flagstaff, Arizona, as shown in Table 2. Figure 1 includes spectra of an M8.5, an L0.5, and an L3.5 dwarf, which covers the range of ultracool dwarf spectral types 
Table 2

Observation Details

\begin{tabular}{|c|c|c|c|c|c|c|c|c|c|c|}
\hline Source & $\begin{array}{l}\text { Epochs } \\
\text { (No.) } \\
(2)\end{array}$ & $\begin{array}{l}\text { Total Time/ } \\
\text { Baseline } \\
\text { ( hr; yr) } \\
\text { (3) }\end{array}$ & $\begin{array}{c}\text { Date } \\
\text { of Obs. } \\
\text { (UT) } \\
(4)\end{array}$ & $\begin{array}{l}\text { Length } \\
\text { of Obs. } \\
(\sim \mathrm{hr}) \\
(5)\end{array}$ & $\begin{array}{c}\text { Exp. } \\
\text { Time } \\
(\mathrm{s} \times \text { coadd }) \\
\quad(6)\end{array}$ & (7) & $\begin{array}{c}\text { Readout } \\
\text { Rate } \\
(\mathrm{MHz}) \\
(8)\end{array}$ & (9) & $\begin{array}{l}\text { Refs. } \\
\text { (No.) } \\
(10)\end{array}$ & $\begin{array}{l}\text { Telescope/ } \\
\text { Inst. } \\
\text { (11) }\end{array}$ \\
\hline \multirow[t]{11}{*}{ LP $349-25 \mathrm{AB}$} & 3 & $64 ; 1.2$ & 2009 Sep 22 & 7.2 & $5 \times 24$ & $I$ & 1 & Conv. & 5 & VATT/GUFI \\
\hline & & & 2009 Sep 26 & 4.0 & $5 \times 24$ & $I$ & 1 & Conv. & 5 & VATT/GUFI \\
\hline & & & 2010 Oct 9 & 4.0 & $5 \times 12$ & $I$ & 1 & Conv. & 5 & VATT/GUFI \\
\hline & & & 2010 Oct 10 & 6.4 & $5 \times 12$ & $I$ & 1 & Conv. & 5 & VATT/GUFI \\
\hline & & & 2010 Oct 11 & 5.2 & $5 \times 12$ & $I$ & 1 & Conv. & 5 & VATT/GUFI \\
\hline & & & 2010 Oct 12 & 5.5 & $5 \times 12$ & $I$ & 1 & Conv. & 5 & VATT/GUFI \\
\hline & & & 2010 Oct 13 & 6.5 & $5 \times 12$ & $I$ & 1 & Conv. & 5 & VATT/GUFI \\
\hline & & & 2010 Oct 14 & 7.0 & $5 \times 12$ & $I$ & 1 & Conv. & 5 & VATT/GUFI \\
\hline & & & 2010 Oct 15 & 6.0 & $5 \times 12$ & $R$ & 1 & Conv. & 4 & VATT/GUFI \\
\hline & & & 2010 Nov 16 & 7.3 & $5 \times 12$ & $I$ & 1 & Conv. & 5 & VATT/GUFI \\
\hline & & & 2010 Nov 27 & 5.0 & $5 \times 12$ & $I$ & 1 & Conv. & 5 & VATT/GUFI \\
\hline \multirow[t]{11}{*}{ 2M J0746AB } & 4 & $62 ; 2$ & 2009 Jan 25 & 6.0 & $25 \times 1$ & $I$ & $\cdots$ & Conv. & 20 & VATT/4K \\
\hline & & & 2009 Jan 26 & 6.8 & $25 \times 1$ & $I$ & $\cdots$ & Conv. & 15 & VATT/4K \\
\hline & & & 2009 Jan 28 & 7.4 & $25 \times 1$ & $I$ & $\cdots$ & Conv. & 19 & VATT/4K \\
\hline & & & 2010 Feb 19 & 4.5 & $5 \times 12$ & $I$ & 1 & Conv. & 5 & VATT/GUFI \\
\hline & & & 2010 Feb 20 & 4.0 & $5 \times 12$ & $I$ & 1 & Conv. & 6 & VATT/GUFI \\
\hline & & & 2010 Nov 13 & 4.6 & $5 \times 12$ & $I$ & 1 & Conv. & 6 & VATT/GUFI \\
\hline & & & 2010 Nov 14 & 5.5 & $5 \times 12$ & $I$ & 1 & Conv. & 5 & VATT/GUFI \\
\hline & & & 2010 Dec 2 & 6.0 & $5 \times 12$ & $I$ & 1 & Conv. & 6 & VATT/GUFI \\
\hline & & & 2010 Dec 12 & 3.0 & $5 \times 12$ & $I$ & 1 & Conv. & 6 & VATT/GUFI \\
\hline & & & 2010 Dec 13 & 6.8 & $5 \times 12$ & $I$ & 1 & Conv. & 6 & VATT/GUFI \\
\hline & & & 2010 Dec 14 & 7.0 & $5 \times 12$ & $I$ & 1 & Conv. & 6 & VATT/GUFI \\
\hline \multirow[t]{8}{*}{ LSR J1835 } & 3 & $33 ; 3$ & $2006 \mathrm{Jul} 17$ & 7.0 & $5 \times 12$ & $I$ & 1 & Conv. & 5 & Loiano/GUFI \\
\hline & & & 2006 Jul 20 & 6.5 & $5 \times 12$ & $I$ & 1 & Conv. & 5 & Loiano/GUFI \\
\hline & & & 2006 Sep 22 & 3.6 & $30 \times 2$ & $I$ & $\ldots$ & $\ldots$ & 10 & USNO/Tek2k \\
\hline & & & 2006 Sep 24 & 3.0 & $30 \times 2$ & $R$ & $\ldots$ & $\ldots$ & 10 & USNO/Tek2k \\
\hline & & & 2009 Jun 11 & 2.2 & $5 \times 12$ & $I$ & 1 & Conv. & 5 & VATT/GUFI \\
\hline & & & 2009 Jun 13 & 4.0 & $5 \times 12$ & $I$ & 1 & Conv. & 5 & VATT/GUFI \\
\hline & & & 2009 Jun 16 & 4.0 & $5 \times 12$ & $I$ & 1 & Conv. & 4 & VATT/GUFI \\
\hline & & & 2009 Jun 30 & 3.0 & $5 \times 12$ & $I$ & 1 & Conv. & 5 & VATT/GUFI \\
\hline \multirow[t]{10}{*}{ TVLM 513} & 6 & $53 ; 5$ & 2006 May 21 & 4.8 & $30 \times 3$ & $I$ & $\cdots$ & $\ldots$ & 6 & VATT/2K \\
\hline & & & 2008 Jun 17 & 6.0 & $60 \times 2.5$ & $I$ & $\cdots$ & $\ldots$ & 10 & USNO/new $2 \mathrm{k}$ \\
\hline & & & 2009 Jun 12 & 3.6 & $5 \times 12$ & $I$ & 1 & Conv. & 5 & VATT/GUFI \\
\hline & & & 2009 Jun 13 & 4.1 & $5 \times 12$ & $I$ & 1 & Conv. & 5 & VATT/GUFI \\
\hline & & & 2009 Jun 16 & 4.0 & $5 \times 12$ & $I$ & 1 & Conv. & 6 & VATT/GUFI \\
\hline & & & 2011 Feb 18 & 3.5 & $5 \times 12$ & $I$ & 1 & Conv. & 6 & VATT/GUFI \\
\hline & & & $2011 \mathrm{Feb} 25$ & 4.3 & $5 \times 12$ & $I$ & 1 & Conv. & 5 & VATT/GUFI \\
\hline & & & 2011 Apr 12 & 7.0 & $5 \times 12$ & $I$ & 1 & Conv. & 6 & VATT/GUFI \\
\hline & & & 2011 May 7 & 8.0 & $25 \times 1$ & $i^{\prime}$ & $\cdots$ & $\ldots$ & 12 & VATT/4K \\
\hline & & & 2011 May 8 & 8.0 & $25 \times 1$ & $i^{\prime}$ & $\cdots$ & $\ldots$ & 12 & VATT/4K \\
\hline \multirow[t]{6}{*}{ BRI 0021} & 3 & $28 ; 1.2$ & 2009 Sep 14 & 4.0 & $5 \times 12$ & $I$ & 1 & Conv. & 1 & VATT/GUFI \\
\hline & & & 2009 Sep 16 & 5.1 & $5 \times 12$ & $I$ & I & Conv. & 1 & VATT/GUFI \\
\hline & & & 2010 Nov 13 & 4.0 & $5 \times 12$ & $I$ & 1 & Conv. & 1 & VATT/GUFI \\
\hline & & & 2010 Nov 14 & 5.5 & $5 \times 12$ & $I$ & 1 & Conv. & 1 & VATT/GUFI \\
\hline & & & 2010 Dec 2 & 5.1 & $5 \times 12$ & $I$ & 1 & Conv. & 1 & VATT/GUFI \\
\hline & & & 2010 Dec 3 & 4.5 & $5 \times 12$ & $I$ & 1 & Conv. & 1 & VATT/GUFI \\
\hline \multirow[t]{2}{*}{ 2M J0036 } & 2 & $10 ; 0.03$ & 2010 Dec 1 & 5.5 & $5 \times 24$ & $I$ & 1 & Conv. & 6 & VATT/GUFI \\
\hline & & & 2010 Dec 13 & 5.0 & $5 \times 24$ & $I$ & 1 & Conv. & 6 & VATT/GUFI \\
\hline
\end{tabular}

Notes. Column 1: campaign source. Column 2: the number of epochs over the course of the campaign. All epochs may contain multiple nights of observations, where these are not always sequential. Column 3: the total amount of hours on target in hours, and the total temporal baseline in years. Column 4: observation dates for each target. Column 5: the length of each observation, as shown in the relevant figures in Section 5. Column 6: the exposure time of each observation, as well as the binning factor used for final data points as shown in this paper. Column 7: the wave band used for a particular observation. Column 8: the readout rate used, in MHz. This column only applies to the GUFI mk.II system. Column 9: the amplifier used. Again, only applicable to GUFI mk.II. Column 10: the number of reference stars used for a given observation. We highlight that since the VATT $4 \mathrm{~K}$ Imager provided a FOV of $\sim 12.5 \times 12.5$, many more reference stars were available when compared to the smaller $\sim 3^{\prime} \times 3^{\prime}$ FOV of GUFI mk.II. Furthermore, there was one available star suitable for effective differential photometry in the case of BRI 0021. Although we could not confirm its stability against another non-varying star in the same field, we chose to use this based on the observations of Martín et al. (2001), who confirmed it as a stable source during their photometric observations (indicated as reference star 1, in Figure 1 of their work). Column 11: telescope and detector used. 
that our observations covered. Typical acquisition parameters are also summarized in Table 2.

Data reduction was carried out using the in-house GUFI L3 Pipeline (Sheehan \& Butler 2008). Standard data reduction techniques were employed where the data were bias subtracted using zero-integration frames and flat-fielded using twilight flatfields. Twilight flat-fields for any given observation consisted of $>100$ median-combined dithered frames taken from a blank part of the sky. Frames were registered and summed in image space to increase the signal-to-noise ratio $(\mathrm{S} / \mathrm{N})$, and differential photometry was carried out on all science data in order to achieve milli-magnitude photometric precision.

The FOVs of the GUFI, the VATT 4K, and the USNO photometers, provide between 1-20 reference stars for a given field. Photometry for all reference stars was also obtained as a measure of their stability in order to ensure that variability was intrinsic to the target star. These stars were chosen on the basis of their stability, position, isolation, the properties of their seeing profiles, and comparable magnitudes and color to that of the target. Photometric apertures (in pixels) which provided the highest $\mathrm{S} / \mathrm{N}$ for the target star were selected for aperture photometry; however, aperture and sky annulus diameters varied from night to night depending on the average seeing conditions, which typically ranged from 0.7 to 1.6 arcsec. Differential photometry was obtained by dividing the target flux by the mean flux of selected reference stars. Although changing seeing conditions can ultimately introduce photometric errors, for all observations we ensured that the photometric parameters remained constant for all stars - this allowed the same fraction of total flux to be observed in the aperture of each source.

\section{ASSESSING PERIODIC VARIABILITY}

In order to detect periodic variability and assess its significance, we used a variety of statistical tests as a means of assessing the validity of any detected periodic signals, and to calculate the associated errors. This assessment was carried out in order of the procedures below. These are well established techniques and so we only briefly explain each in the relevant sections-we refer the reader to the references therein for more in-depth discussions.

\subsection{Lomb-Scargle Periodogram}

The first method used for the detection of periodic signals was the calculation of the Lomb-Scargle (LS) periodogram (Lomb 1976; Scargle 1982), a technique which is effective for unevenly spaced data. The LS periodogram uses the discrete Fourier transform, which provides power spectra that are analyzed for significant peaks - corresponding to possible periodic variability. In the case of an arbitrary (unevenly) sampled dataset, the LS periodogram is calculated by the following (where the power spectrum $P$, is a function of angular frequency $\omega=2 \pi f>0$ ):

$$
\begin{aligned}
P(\omega)= & \frac{1}{2 \sigma_{\mathrm{var}}^{2}} \frac{\left[\sum_{i}\left(h_{i}-\bar{h}\right) \cdot \cos \cdot \omega\left(t_{i}-\tau\right)\right]^{2}}{\sum_{i} \cos ^{2} \cdot \omega\left(t_{i}-\tau\right)} \\
& +\frac{\left[\sum_{i}\left(h_{i}-\bar{h}\right) \cdot \sin \cdot \omega\left(t_{i}-\tau\right)\right]^{2}}{\sum_{i} \sin ^{2} \cdot \omega\left(t_{i}-\tau\right)},
\end{aligned}
$$

where $\tau=\tan (2 \cdot \omega \cdot t)=\left(\sum_{i} \sin \cdot 2 \cdot \omega t_{i} / \sum_{i} \cos \cdot 2 \cdot \omega t_{i}\right)$, each consecutive data point is $h_{i}$, the mean of the data is $\bar{h}$, and the variance is $\sigma_{\mathrm{var}}^{2}$.
In this work, we selected a range of peaks corresponding to possible periodic solutions as provided by the technique above. We inspected these solutions by phase connecting raw light curves to a given solution, and assessed their level of agreement in phase. We rule out solutions $>0.25$ out of phase. In addition, we overplotted LS power spectra for different epochs, investigated which peaks were in greatest agreement, and then compared these to the strongest phase folded solutions.

\subsection{Phase Dispersion Minimization}

We also investigated the phase dispersion minimization (PDM) technique as outlined by Stellingwerf (1978), as a second statistical tool. Stellingwerf (1978) describes the PDM method as a least squares fit (LSF) approach where a fit is calculated by using the mean curve of the data, controlled by the mean of each bin (which can be specified in the algorithm), and the period that produces the least datapoint scatter, or "PDM theta statistic" $(\Theta)$, about this computed mean, is the most likely solution.

The PDM technique phase folds selected light curves to a range of periods, and their significance is calculated. It is useful for data sets with large gaps, and furthermore, it is insensitive to the light curve's shape and therefore makes no assumptions with regard to the morphology. The routine also includes a Monte Carlo test, used for assessing the statistical significance of the detected $\Theta$ minima. It computes this by randomizing the data point order, which removes the signal component. We repeated this for $10^{5}$ trials in order to cover a significant distribution of $\Theta$ values due to noise. ${ }^{9}$ Similar to the LS technique above, it is possible for many periodic solutions to present themselves due to aliasing - a consequence of gaps in the data. We take the minimum $\Theta$ from the PDM analysis, and compare it to the highest peak in the power spectra of the LS.

\subsection{Amplitude Variability Analysis}

We established the peak to peak (PtP) amplitude variability of the target light curves by means of sinusoid fitting and the $\chi^{2}$ technique, where the phase and amplitude of a sinusoidal function were varied, and then the $\chi^{2}$ minimization was performed. We took this amplitude (which is a PtP measure of the change of relative flux) as $\mathrm{PtP}_{\mathrm{tar}}$. This is a weighted assessment and so does not treat each data point equally; the error in each point is utilized in the calculation of the best-fit amplitude and the error in the amplitude.

The corresponding reference star variability was found via the standard deviation of its light curve $\left(\sigma_{\text {ref }}\right)$. We plotted each reference star flux against all others to ensure that each chosen selected reference star was non-variable. Although variability can statistically be detected if the standard deviation is only fractionally larger than the error in the light curve's relative magnitude, the periodic variability detected in our target data is categorically present in each epoch, where the variability is clearly above the standard deviation of the reference star relative flux. Furthermore, different sets/combinations of reference stars were used as a "sanity check" to confirm that the signal was indeed intrinsic to the target star.

\subsection{Photometric Error Estimation}

The photometric error analysis was calculated via the iraf.phot ${ }^{10}$ routines in all target and reference star light curves.

\footnotetext{
9 We cite Stellingwerf (1978) for the PDM routines, but refer to his latest work at http://www.stellingwerf.com/.

${ }^{10}$ Image Reduction and Analysis Facility-http://iraf.noao.edu/.
} 
An estimation of the error in the relative magnitude $\left(\delta m_{\star}\right)$ of the target star was found as follows:

$$
\left(\delta m_{\star}\right)^{2}=\left(\delta_{\text {target }}\right)^{2}+\left(\frac{1}{M F_{i}}\right)^{2} \sum_{n}^{M} F_{n}^{2}\left(\delta m_{n}\right)^{2},
$$

where $M$ is the number of reference stars, $F_{i}$ is the mean flux of the reference stars, $F_{n}$ is the flux of the $n_{t h}$ reference star, and $\delta m_{n}$ is the magnitude error in the $n_{t h}$ reference star. This error in magnitude was then converted to an error in flux. We show these error bars on each data point in each light curve. This method takes both formal and informal errors such as flat-fielding and residual fringing (Section 4.5) into account — which are difficult to assess in separate cases.

In addition to the formal and informal errors, we also identify detector response at nonlinear regimes as a source of potential error. We avoid such nonlinear effects by keeping exposure times low enough to maintain levels to no greater than $75 \%$ of pixel saturation. After taking these effects into account, we move to calculating the period uncertainty.

\subsection{Fringing}

Fringing is an optical effect or disturbance in the thinnedsubstrate of back-illuminated CCDs and is present as a result of $\mathrm{OH}$ spectral emission in the atmosphere. Fringing interferes at red/NIR wavelengths and since the CCD's substrate becomes transparent at these wavelengths, any waveband that approaches the NIR is more susceptible to these fringing effects. It varies as a function of amplitude, but not position. Since the amplitude variations expected in these ultracool dwarf targets are of the order of milli-magnitudes, it is important to remove these additive effects if the amplitude variations due to fringing are potentially greater than the target star differential light curves. The standard procedure for this correction includes the creation of a fringing template from well sampled median-combined deep sky frames containing only the fringing pattern, normalizing this template to each individual frame's sky background level and then subtracting it. We obtained dithered sky frames for all Sloan $i^{\prime}$ and $I$-band observations to allow for fringe removal if necessary. We also took dome flat-fields which contain none of these atmospheric effects, in addition to twilight flat-fields. We conducted tests to investigate the effect of this artifact on each consecutive data set, and if the amplitude of the fringing pattern was varying at a greater level than that of the mean sky background, it was removed.

\subsection{Phase Connecting and Period Uncertainty Estimation}

We achieve an accurate enough period of rotation for the M9 dwarf TVLM 513 to phase connect its $\sim 5 \mathrm{yr}$ baseline. We could not phase connect any other target, and thus the procedure outlined here applies to TVLM 513 only. Standard phase connection techniques were employed whereby the period accuracy increased as epochs were successfully phase connected, enabling an assessment of the correlation of the peak of each phase solution. This allowed us to combine data from two different epochs, if the period from a single epoch could be calculated with sufficient accuracy, such that the rotational phase of the second epoch was unambiguous - in this work we define this threshold to be $\delta \phi<0.25$.

In order to assess the period error for all other targets, we overplotted the LS power spectra period range with a Gaussian profile, and calculated the FWHM. In this way, we estimate $1 \sigma$ errors on the period uncertainty $(\delta P)$ for these targets. Since the $\mathrm{FWHM}=2 \sqrt{2 \ln 2} \sigma=2.35482 \sigma, \delta P$ is therefore defined as:

$$
\delta P=\frac{\mathrm{FWHM}}{2.35482} .
$$

We find that the uncertainty range calculated for each target for the best-fit period of rotation, allowed other possible solutions within this range to be phased together within epochs. The $\chi^{2}$ test outlined in the previous section also provided a measure of the period error per given fit. Other authors have also established various means of assessing the error in the frequency of a signal, e.g., Schwarzenberg-Czerny (1991); and Akerlof et al. (1994). These techniques can largely rely on data uniformly sampled in time. Thus, similar to the $\chi^{2}$ fitting, they were effective in calculating an error for a single observation, but not for unevenly spaced baselines.

\section{RESULTS}

\subsection{General Results}

We report periodic variability for five of the six radio-detected dwarfs in the sample. Our assessment of the PtP amplitude variations for each target are shown in Table 3, and the periodic variability results are shown in Table 4 . The properties of this periodicity is generally consistent for all dwarf spectral types, where we detect periodic sinusoidal variability over timescales of years. All dwarfs exhibit changes in amplitude throughout the campaign, which we discuss in Section 6.

In the following subsections, we outline general results and variability analysis of each target, as well as light curve and photometric properties. All confirmed periods in these data were detected to significance values exceeding $5 \sigma$. The target results are shown in Figures $2-7$, and the variability analysis for each is shown in Figure 8. We discuss the possibilities for the cause of this periodic variability in Section 6.

\subsection{Binary Dwarfs$$
\text { 5.2.1. LP 349-25 }
$$

We detect the binary as a periodically varying source in VATT $R$ band and $I$ band, which we report as the first detected optical variability of this system. The primary period of 1.86 $\pm 0.02 \mathrm{hr}$ is present in each band and varying with a $\mathrm{PtP}_{\text {tar }}$ range of $0.44 \%-1.42 \%$ in the $I$ band, and $1.96 \%$ in the $R$ band (single observation), as shown in Table 3 and Figure 2. The LS periodogram and PDM statistical analysis is shown at the end of the section in Figure 8. Mean $\sigma_{\text {ref }}$ were calculated to be $\sim 0.30 \%$ and $\sim 0.68 \%$ in the $I$ band and $R$ band, respectively. We see larger $\sigma_{\text {ref }}$ in the $R$ band due to intermittently poor seeing. It is difficult to assess the amplitude ratios between each band, since the amplitude level in the $I$ band is varying at different levels during observations (Table 3 ). Furthermore, we did not obtain simultaneous $R$-band and $I$-band data.

Despite the consistency of the primary periodic component throughout the observations, we observe some aperiodic variations in addition to significant variations in amplitude during some I-band observations (e.g., Figure 2: 2010 October 10, 11, and 13). We do not image each component of the binary as a single point source in these observations. Therefore, the detected sinusoidal periodicity in our data is due to the combined flux of both binary members. We observe unusual behavior for some of the 2010 October epoch, where the periodic signal appears to 
Table 3

Peak to Peak Amplitude Variability and Photometric Error Analysis of Sample

\begin{tabular}{|c|c|c|c|c|c|c|}
\hline Source & $\begin{array}{c}\text { Date of Obs. } \\
\text { (UT) }\end{array}$ & Band & $\begin{array}{l}\mathrm{PtP}_{\operatorname{tar}} \\
(\%)\end{array}$ & $\begin{array}{c}\text { Phot. Error } \\
(\%)\end{array}$ & $\begin{array}{c}\mathrm{PtP}_{\text {tar }} \text { Range } \\
(\%)\end{array}$ & $\begin{array}{c}\text { Mean } \sigma_{\text {ref }} \\
(\%)\end{array}$ \\
\hline (1) & (2) & (3) & (4) & (5) & (6) & (7) \\
\hline \multirow[t]{10}{*}{ LP 349-25AB } & 2009 Sep 22 & $I$ & 0.48 & 0.15 & $0.44-1.42(I) ; 1.96(R)$ & $0.30(I) ; 0.68(R)$ \\
\hline & 2009 Sep 26 & $I$ & 1.42 & 0.21 & & \\
\hline & 2010 Oct 9 & $I$ & 1.04 & 0.21 & & \\
\hline & 2010 Oct 10 & $I$ & 0.90 & 0.22 & & \\
\hline & 2010 Oct 12 & $I$ & 0.94 & 0.21 & & \\
\hline & 2010 Oct 13 & $I$ & 0.90 & 0.15 & & \\
\hline & 2010 Oct 14 & $I$ & 0.58 & 0.15 & & \\
\hline & 2010 Oct 15 & $R$ & 1.96 & 0.53 & & \\
\hline & 2010 Nov 16 & $I$ & 1.12 & 0.23 & & \\
\hline & 2010 Nov 27 & $I$ & 0.92 & 0.15 & & \\
\hline \multirow[t]{10}{*}{ 2M J0746AB } & 2009 Jan 25 & $I$ & 0.40 & 0.21 & $0.40-1.52(I)$ & $0.36(I)$ \\
\hline & 2009 Jan 28 & $I$ & 0.78 & 0.24 & & \\
\hline & 2010 Feb 19 & $I$ & 1.26 & 0.27 & & \\
\hline & 2010 Feb 20 & $I$ & 1.32 & 0.30 & & \\
\hline & 2010 Nov 13 & $I$ & 1.18 & 0.31 & & \\
\hline & 2010 Nov 14 & $I$ & 1.04 & 0.33 & & \\
\hline & 2010 Dec 2 & $I$ & 0.68 & 0.25 & & \\
\hline & 2010 Dec 12 & $I$ & 1.38 & 0.29 & & \\
\hline & 2010 Dec 13 & $I$ & 1.52 & 0.32 & & \\
\hline & 2010 Dec 14 & $I$ & 0.96 & 0.34 & & \\
\hline \multirow[t]{6}{*}{ LSR J1835 } & 2006 Jul 17 & $I$ & 1.08 & 0.12 & $1.02-1.46(I) ; 1.62(R)$ & $0.33(I) ; 0.68(R)$ \\
\hline & 2006 Jul 20 & $I$ & 1.02 & 0.13 & & \\
\hline & 2006 Sep 22 & $I$ & 1.46 & 0.43 & & \\
\hline & 2006 Sep 24 & $R$ & 1.62 & 1.20 & & \\
\hline & 2009 Jun 11 & $I$ & 1.24 & 0.12 & & \\
\hline & 2009 Jun 30 & $I$ & 1.36 & 0.18 & & \\
\hline \multirow[t]{10}{*}{ TVLM 513} & 2006 May 21 & $I$ & 0.82 & 0.42 & $0.56-1.20(I) ; 0.92-0.96\left(i^{\prime}\right)$ & $0.34(I) ; 0.36\left(i^{\prime}\right)$ \\
\hline & 2008 Jun 17 & $I$ & 0.66 & 0.53 & & \\
\hline & 2009 Jun 12 & $I$ & 0.56 & 0.30 & & \\
\hline & 2009 Jun 13 & $I$ & 0.72 & 0.23 & & \\
\hline & 2009 Jun 16 & $I$ & 1.14 & 0.25 & & \\
\hline & 2011 Feb 18 & $I$ & 1.20 & 0.32 & & \\
\hline & 2011 Feb 25 & $I$ & 0.70 & 0.32 & & \\
\hline & 2011 Apr 12 & $I$ & 0.76 & 0.31 & & \\
\hline & 2011 May 7 & $i^{\prime}$ & 0.96 & 0.27 & & \\
\hline & 2011 May 8 & $i^{\prime}$ & 0.92 & 0.26 & & \\
\hline \multirow[t]{6}{*}{ BRI 0021} & 2009 Sep 14 & $I$ & 1.10 & 0.33 & $0.52-1.58(I)$ & $0.37(I)$ \\
\hline & 2009 Sep 16 & $I$ & 0.90 & 0.32 & & \\
\hline & 2010 Nov 13 & $I$ & 0.72 & 0.32 & & \\
\hline & 2010 Nov 14 & $I$ & 1.58 & 0.31 & & \\
\hline & 2010 Dec 2 & $I$ & 0.68 & 0.32 & & \\
\hline & 2010 Dec 3 & $I$ & 0.52 & 0.35 & & \\
\hline \multirow[t]{2}{*}{ 2M J0036 } & 2010 Dec 1 & $I$ & 2.20 & 0.82 & $1.98-2.20(I)$ & $1.0(I)$ \\
\hline & 2010 Dec 13 & $I$ & 1.98 & 1.11 & & \\
\hline
\end{tabular}

Notes. Column 1: campaign source. Column 2: date of observation in UT. Column 3: waveband used. Column 4: peak to peak (PtP) amplitude variability as measured by the $\chi^{2}$ test. Column 5: mean photometric error per data point for a given night as calculated by the iraf.phot routines. This is outlined in Section 4.4. Column 6: peak to peak amplitude variability range of target light curves, shown in the $I$ band or Sloan $i^{\prime}$, and in the $R$ band, for selected targets. Both $R$-band results are from single observations. Column 7: standard deviation of non-variable reference star light curve in the $R$ band, Sloan $i^{\prime}$, and the $I$ band (mean standard deviation of all reference stars used in each case).

move in and out of phase during single observations of $\sim 8 \mathrm{hr}$; we give examples of this in Section 6.4.

Finally, the radii estimates of Dupuy et al. (2010) and individual rotation velocity measurements of Konopacky et al. (2012) infer maximum rotation periods of $\sim 2.65 \mathrm{hr}$ and $\sim 1.67 \mathrm{hr}$ for each component, respectively. Therefore, we have a tentative case to argue in favor of LP $349-25 B$ as the periodically varying source in $R$ - and $I$-band wavelengths. However, the radii estimates of Konopacky et al. (2010) are at odds with those derived in this work as well as the estimates of Dupuy 
Table 4

Confirmed Optical Periodic Variability in Radio-detected Ultracool Dwarf Sample

\begin{tabular}{lcccccc}
\hline \hline Parameter & LP 349-25B & 2M J0746A & LSR J1835 & TVLM 513 & BRI 0021 & 2M J0036 \\
\hline (1) Period (hr) & $1.86 \pm 0.02$ & $3.32 \pm 0.15$ & $2.845 \pm 0.003$ & $1.95958 \pm 0.00005$ & $?(\sim 5)$ & $\sim 3.0 \pm 0.7$ \\
(2) LS period (hr) & 1.86 & 3.32 & 2.845 & 1.95958 & $\ldots$ & 2.5 \\
(3) PDM period (hr) & 1.86 & 3.32 & 2.844 & 1.95959 & $\ldots$ & $\ldots$ \\
(4) References & 1 & 1,2 & 1 & 1,3 & 1,3 \\
\hline
\end{tabular}

Notes. Row 1: period of rotation and associated error as calculated in Section 4. Row 2: Lomb-Scargle periodogram periods: the quoted periods are those which were determined to be the most likely solution based on the correlation of the highest peaks in all periodograms (all data combined and individual epochs). Row 3: phase dispersion minimization periods: the PDM periods shown here represent the lowest $\Theta$ statistic calculated by the PDM routines, as is shown in Section 5.

References. (1) This work; (2) Harding et al. (2013); (3) Lane et al. (2007); TVLM 513 originally published as $~ 1.96$ hr, $2 \mathrm{M}$ J0036 published as $\sim 3 \mathrm{hr}$.

et al. (2010). This modeling and the association of the $1.86 \mathrm{hr}$ period with LP 349-25B are discussed later in Section 6.5.

\subsubsection{MASSW J0746425+200032}

The periodic variability of $2 \mathrm{M} \mathrm{J} 0746 \mathrm{AB}$ has recently been discussed by Harding et al. (2013), who use this rotation period to infer the coplanarity of the spin axis and orbital plane. We include a discussion of the variability here again for completeness. Although we do not resolve each component of the binary as a point source, we report optical periodic

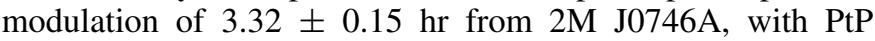
amplitude variability of $\mathrm{PtP}_{\text {tar }} \sim 0.40 \%-1.52 \%$ in the VATT $I$-band (Figure 3 ), and a mean reference star standard deviation of $\sigma_{\text {ref }} \sim 0.36 \%$.

It appears that this optical periodic variability originates from the other component to that producing the radio emissionreported by Berger et al. (2009) where the binary exhibited periodic bursts of radio emission of $2.07 \pm 0.002 \mathrm{hr}$. The estimated radii of $\sim 0.99 \pm 0.03 R_{J}$ and $\sim 0.96 \pm 0.02 R_{J}$ (Harding et al. 2013), in addition to the well established $v$ sin $i$ measurements (Konopacky et al. 2012), infer maximum rotation periods for $2 \mathrm{M} \mathrm{J} 0746 \mathrm{~A}$ and $2 \mathrm{M} \mathrm{J} 0746 \mathrm{~B}$ of $\sim 4.22 \mathrm{hr}$ and $\sim 2.38 \mathrm{hr}$, respectively. Therefore, the period of $3.32 \pm$ $0.15 \mathrm{hr}$ likely emanates from 2M J0746A, whereas Berger et al. (2009) found emission from the secondary-2M J0746B. This optical periodicity is categorically present in all epochs as shown in Figure 3, and thus is that of the slower rotating binary dwarf.

\subsection{Single Dwarf Systems}

\subsubsection{LSR J1835+3259}

We determined a photometric period of $2.845 \pm 0.003 \mathrm{hr}$ in VATT $I$ band, consistent with the VLA radio observations of Hallinan et al. (2008), who report periodic pulses of $2.84 \pm$ $0.01 \mathrm{hr}$. This optical period is newly reported in this work, which was conducted between 2006 July and 2009 June with the GUFI mk.I and mk.II systems (Figure 4). We also obtained $R$-band data from the $1.55 \mathrm{~m}$ USNO telescope and detected a periodicity of $\sim 2.84 \mathrm{hr}$. The weather for this observation was very poor; however, it appears that LSR J1835 has larger $R$-band PtP amplitude variability than the $I$-band-similar to LP 349-25. These data exhibit long-term stable periodic sinusoidal variability with a $\mathrm{PtP}_{\text {tar }}$ range of $1.02 \%-1.46 \%$ in the $I$ band and $1.62 \%$ in the $R$ band. The standard deviation of the selected reference stars in each band were $\sigma_{\text {ref }} \sim 0.33 \%$ and $\sim 0.68 \%$, respectively. Furthermore, the calculated period supports the rotational velocity estimate of $v \sin i \sim 50 \pm 5 \mathrm{~km} \mathrm{~s}^{-1}$ (Berger et al. 2008b) and radius estimate of $\geqslant 0.117 \pm 0.012 R_{\odot}$
(Hallinan et al. 2008), which implies a high inclination angle of $\sim 90^{\circ}$ for the system. These data also appear to be in phase based on this period of $2.845 \pm 0.003 \mathrm{hr}$ during constituent epochs. However, we do not achieve a high enough period accuracy in order to phase connect the $\sim 3$ yr temporal baseline. We show the statistical analysis for this target in Figure 8. An example of reference star stability is also shown in red in Figure 4, bottom right.

\subsubsection{TVLM 513-46546}

We confirm periodic variability of $1.95958 \pm 0.00005 \mathrm{hr}$, with a PtP amplitude variability range of $\mathrm{PtP}_{\text {tar }} \sim 0.56 \%-1.20 \%$ in the VATT $I$-band and $\mathrm{PtP}_{\mathrm{tar}} \sim 0.92 \%-0.96 \%$ in Sloan $i^{\prime}$. The morphology of the light curves are generally consistent for both wavebands throughout the campaign, with a mean $\sigma_{\text {ref }}$ of $I: \sim 0.34 \%$ and $i^{\prime}: \sim 0.36 \%$. The larger PtP amplitude variations for some observations are shown in Table 3. This period once again supports previous studies from Hallinan et al. (2006, 2007), Lane et al. (2007), Berger et al. (2008a), and Littlefair et al. (2008), and a clear indication that the photometric I-band periodic variability appears to be stable over timescales of up to $5 \mathrm{yr}$ in this case. It is also consistent with the radius, $v \sin i$, and inclination angle estimates outlined in Hallinan et al. (2008). The calculated $\mathrm{PtP}_{\mathrm{tar}}$ in the $I$ band is lower than the reported PtP amplitude variability of Lane et al. (2007). However, the $i^{\prime}$ variability is much higher than that observed by Littlefair et al. (2008), who detect $\mathrm{PtP}_{\mathrm{tar}}$ of only $\sim 0.15 \%$ in their data. Light curves from each of the six epochs are shown in Figure 5 and the LS periodogram and PDM analysis is shown in Figure 8. In Section 6.2, we show phase connected light curves over the $5 \mathrm{yr}$ baseline in order to investigate the target's phase stability-this study directly investigates the positional stability of the stellar feature responsible for the periodicity. By phase connecting the total baseline of TVLM 513, we were able to establish a period to a much greater accuracy than other targets where phase connection was not possible, due to limited phase coverage.

\subsubsection{BRI 0021-0214}

We report possible photometric VATT $I$-band periodic variability with $\mathrm{PtP}_{\text {tar }}$ of $\sim 0.52 \%-1.58 \%$, and $\sigma_{\text {ref }}$ of $\sim 0.37 \%$, which is shown in Figure 6 . We note that due to $\sim 3^{\prime} \times 3^{\prime}$ FOV of GUFI, there was only one suitable reference star used for differential photometry. This star was selected as a suitable candidate on the basis of its observed stability compared to the target star, during the $I$-band observations of BRI 0021 by Martín et al. (2001). They identify possible periodicity of $\sim 4.8 \mathrm{hr}$ and $\sim 20 \mathrm{hr}$, respectively. We do not have sufficient temporal coverage to effectively assess the presence of a $\sim 20 \mathrm{hr}$ period. Although there 

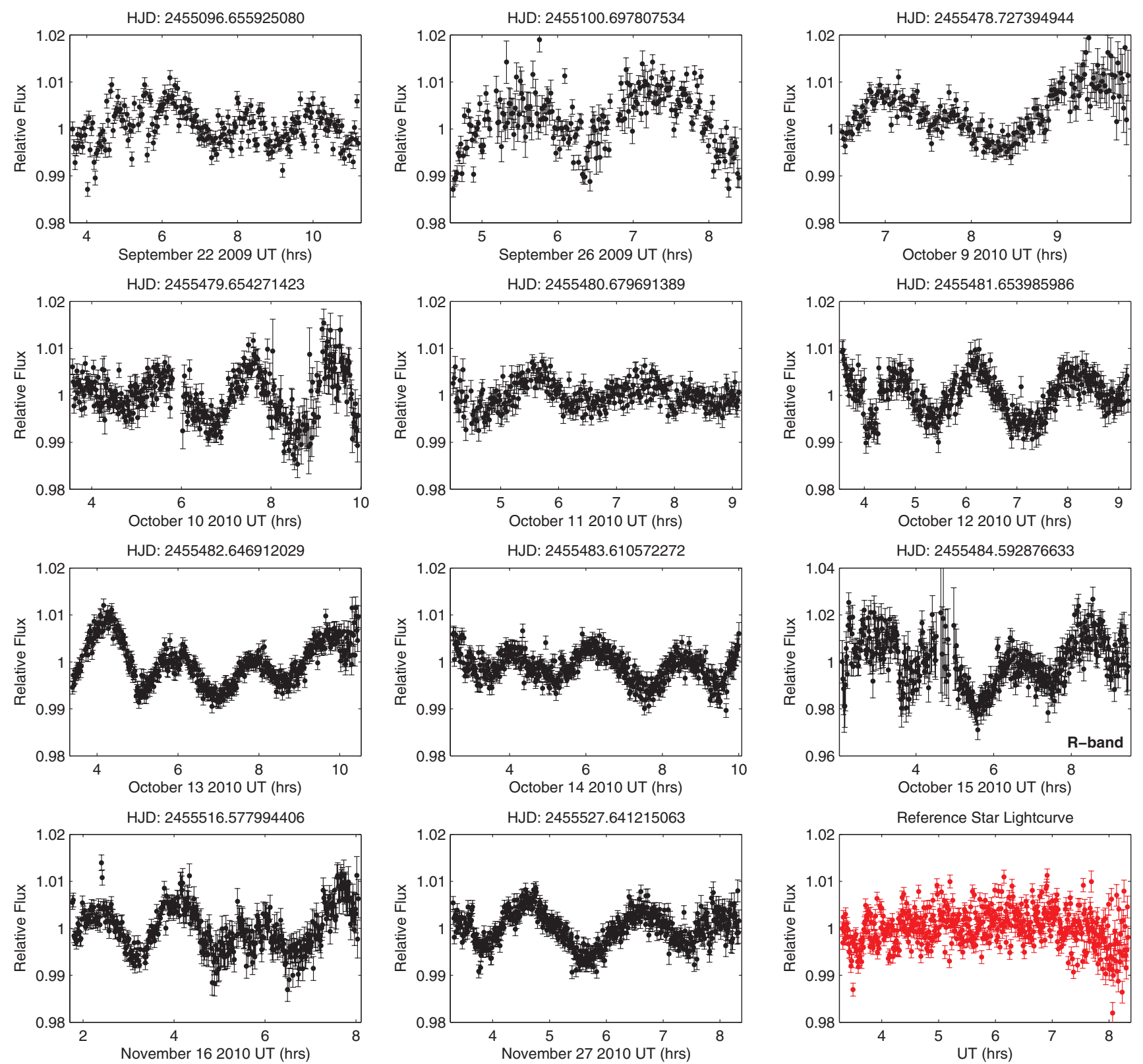

Figure 2. LP 349-25: photometric light curves showing relative flux (y-axis) vs. UT dates and times ( $x$-axis). The HJD time above each figure denotes the start-point of each observation. It is important to note that the $x$-axis range is not the same for each plot, since observations were of different lengths. All data in this figure was taken in the VATT $I$ band ( 7200-9100 $\AA$ ), with the exception of 2010 October 15 UT which was taken in the VATT $R$ band $(\sim 5600-8800 \AA)$ - this is marked on the relevant light curve. Note the difference in scale on the $y$-axis for the $R$-band labeled plot. We detect periodic variability that shows a persistent period of $1.86 \pm 0.02 \mathrm{hr}$ over $\sim 1.2$ yr of observations. These data exhibit changes in amplitude in the $I$ band during consecutive nights (e.g., October 10, 11, 13: $\sim 0.44 \%-1.42 \%$ ), as well as some aperiodic variations observed during some observations (e.g., October 9). The $R$-band light curve exhibits larger peak to peak amplitude variations of $1.96 \%$; the second $R$-band peak in the signal was an interval of poor weather conditions (thin cloud) shown clearly by an increase in the photometric error measurements. The 2009 September epoch was also subject to poor weather conditions (intermittent cloud \& thin cloud throughout), and was therefore binned by a factor of two compared to the other data. Photometric error bars are applied as outlined in Section 4.4. Bottom right: we selected a reference star at random, and plotted its raw flux against the mean raw flux of all other reference stars used in the field. This is used as an example of reference star stability compared to target variability. We note that this light curve is an example of one night only, however we used the same reference stars for all epochs in a given band. The mean reference star variability for all reference stars used in this campaign is shown in Table 3.

(A color version of this figure is available in the online journal.)

is evidence in our statistical analysis of periods between $4-7 \mathrm{hr}$, we do not sample the rotational phase of the object enough to confirm a solution. Since we only have one reference star as a comparison source $\left(00^{\mathrm{h}} 24^{\mathrm{m}} 23^{\mathrm{s}} 735,-01^{\circ} 59^{\prime} 06^{\prime \prime} \cdot 27\right)$, its stability cannot be independently assessed in this case. Interestingly, the possible solutions of $\sim 4-7 \mathrm{hr}$ are in violation with the current $v \sin i$ estimates of $\sim 34 \mathrm{~km} \mathrm{~s}^{-1}$ found by Mohanty \& Basri (2003) — which indicate a maximum period for this system of $\sim 3.59 \mathrm{hr}$. This indicates that the radius of the dwarf could be underestimated if a periodic signal $>3.59 \mathrm{hr}$ is present. Further (larger FOV) observations with greater temporal coverage on a given night are needed to constrain and qualitatively confirm this result.

\subsubsection{MASS J00361617+1821104}

We confirm sinusoidal periodic variability of $3.0 \pm 0.7 \mathrm{hr}$ with $\mathrm{PtP}_{\mathrm{tar}}$ of $1.98 \%-2.20 \%$ in the optical VATT $I$ band. 

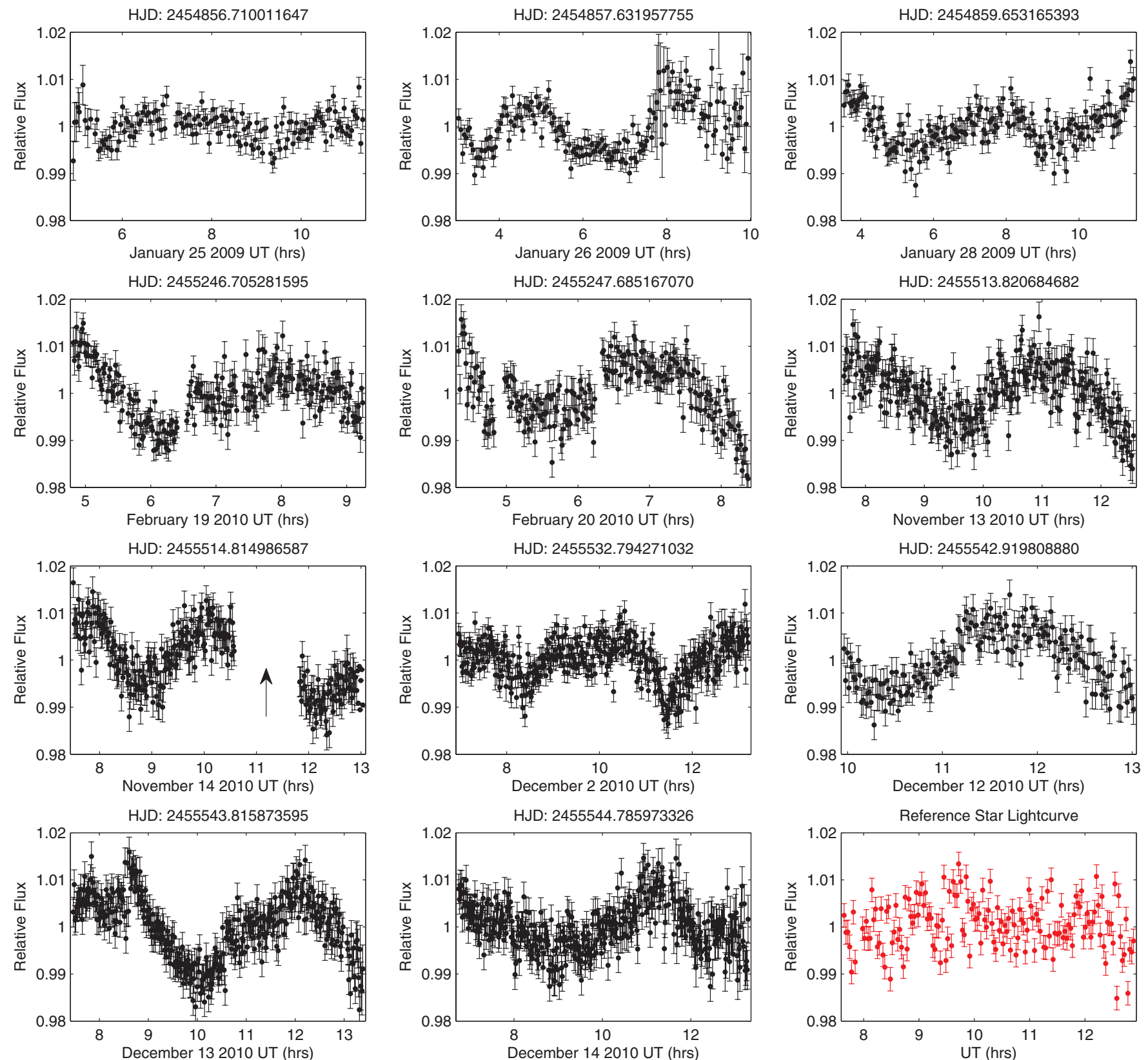

Figure 3. 2MASS J0746+2000: photometric light curves first reported by Harding et al. (2013), and included here for completeness to investigate emission morphology and behavior. Again, UT dates and times are marked on each light curve's $x$-axis along with HJD time above each figure (start-point of each observation). These data were taken in the VATT $I$ band $(\sim 7200-9100 \AA)$ over a $\sim 2$ yr baseline. We report periodic variability for one component of the binary, with a period of $3.32 \pm 0.15 \mathrm{hr}$. The peak to peak amplitude variations throughout the observations varies from $\sim 0.40 \%-1.52 \%$. We note that 2009 January 25 and 26 were taken during deteriorating weather conditions (thin cloud and high winds) and were therefore binned by a factor of two compared to other data. The arrow marked on the 2010 November 14 light curve points to an interval of complete cloud cover, therefore these data were removed. Photometric error bars are applied to each data point as before. Bottom right: as before, an example reference star light curve to illustrate the stability of the chosen reference stars as compared to the target star variability. The mean reference star variability for all reference stars used in this campaign for 2M J0746 is shown in Table 3.

(A color version of this figure is available in the online journal.)

Although these data were obtained under extremely poor seeing conditions on both nights of observation, the range of periods within the calculated error matches the $\sim 3 \mathrm{hr}$ periodicity found by the photometric measurements of Lane et al. (2007) and the radio measurements of Berger et al. (2005) and Hallinan et al. (2008). We note that the observed $\mathrm{PtP}_{\mathrm{tar}}$ is larger than that of other I-band data in this work. We show the differential light curves in Figure 7, and the analysis of these in Figure 8.

\section{DISCUSSION}

\subsection{Source of the Periodicity: The Optical-Radio Correlation?}

A large number of surveys have been carried out to search for evidence of optical variability in ultracool dwarfs. In this work, we consider only late-M to early-to-mid-L dwarfs. Beyond this point, it is clear that the variability has predominantly been associated with dust-related effects (Artigau et al. 2009; Radigan et al. 2012). To date, 182 ultracool dwarfs in this spectral 

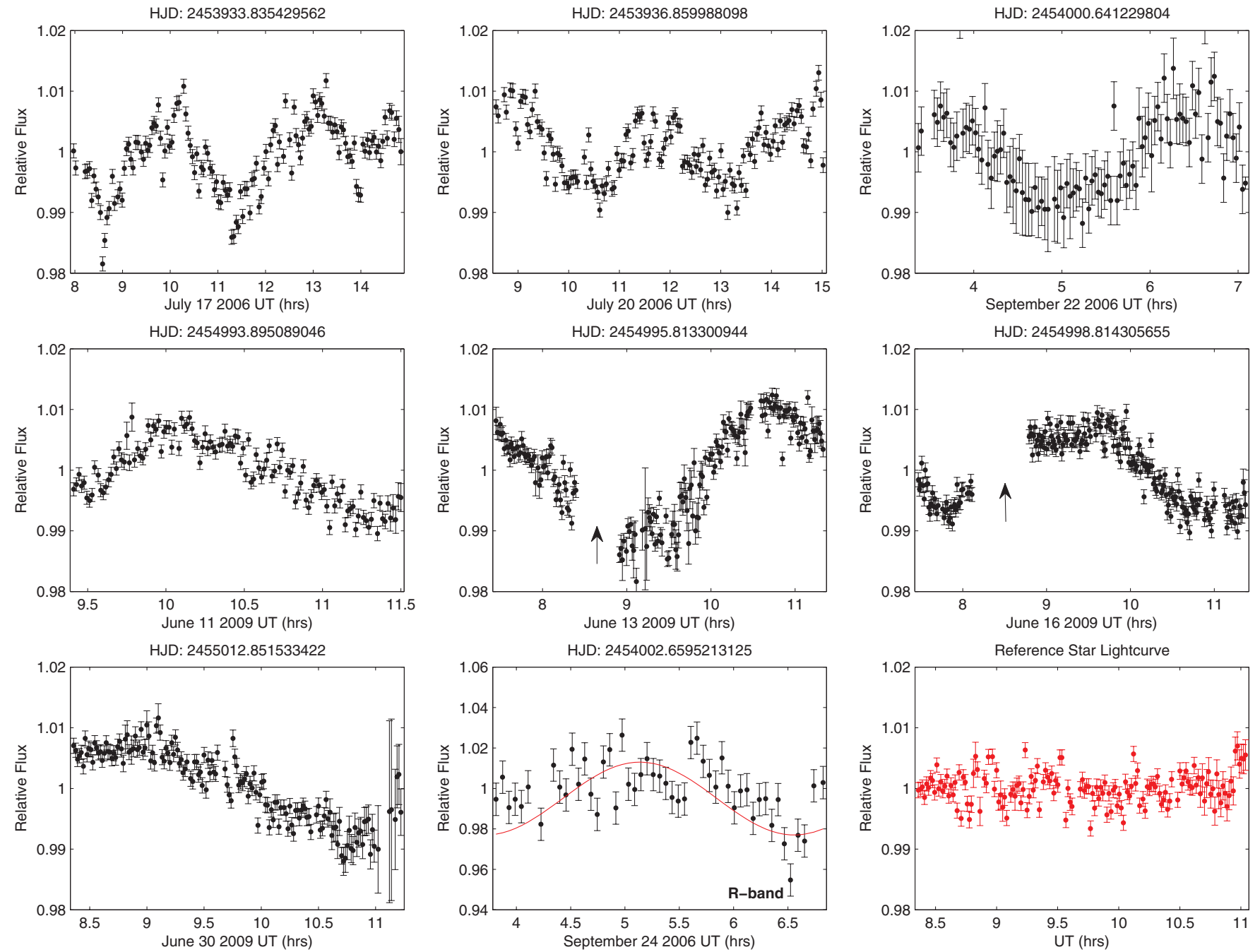

Figure 4. LSR J1835+3259: we report a photometric period of rotation of $2.845 \pm 0.003 \mathrm{hr}$ at $I$-band wavelengths ( $7000-11,000 \AA)$ using the GUFI photometer. These data were taken over a $\sim 3$ yr baseline, where the 2006 July epoch was taken as test data for the GUFI mk.I system. We also observed the dwarf in the $R$ band ( $\sim 5600-8800 \AA$ ) using the USNO on 2006 September 24 and 25 UT. However, the seeing on both nights was very poor. Here, we show a binned data set, marked with an $R$-band label, from 2006 September 24 UT. We overplot a model sinusoidal fit (red) to a period of $2.845 \mathrm{hr}$. The period of rotation of $2.845 \pm 0.003 \mathrm{hr}$ matches the periodic pulses reported by Hallinan et al. (2008), who also attributed this periodicity to the dwarf's rotation. The arrows shown in June 13 and 16 mark data gaps due to this object's passing too close to the zenith for the telescope's Alt-Az tracking. Once again we show a reference star light curve (bottom right) to illustrate the variability of the target with respect to a non-variable source. Although we have a $\sim 3 \mathrm{yr}$ baseline, we do not achieve an accurate enough period to phase connect the 2006 and 2009 epochs.

(A color version of this figure is available in the online journal.)

range $(\geqslant$ M6-L5) have been studied for optical variability, where there has only been $\sim 30 \%-40 \%$ of confirmed variability (Tinney \& Tolley 1999; Bailer-Jones \& Mundt 1999, 2001; Gelino et al. 2002; Clarke et al. 2002a; Koen 2003, 2005, 2012; Koen et al. 2004; Rockenfeller et al. 2006a; Maiti 2007, and references therein). In many cases, these studies have yielded low variability detection rates, or tentative detections with low significance (Koen 2003; Enoch et al. 2003; Koen et al. 2004; Maiti 2007; Goldman et al. 2008). Others have found more promising statistically significant detection rates where the variability was clearly detected above the noise-floor (Bailer-Jones \& Mundt 2001; Gelino et al. 2002; Rockenfeller et al. 2006a). Considering the spectral range in our survey $(\geqslant$ M7.5-L3.5) compared to this same range in the above studies of late-M and early-to-mid-L dwarfs, less than 5\% of objects studied have confirmed periodic variability consistent with the rotation period (Clarke 2002b; Koen 2003, 2006, 2011; Rockenfeller et al. 2006a; Lane et al. 2007).
Our study has confirmed periodic optical variability for five out of six radio active dwarfs, with a tentative detection of similar behavior in the sixth; the latter case is limited by poor sampling of the rotational phase of the object. However, a direct comparison to a large fraction of the above work will show that our sensitivities are much higher for detecting periodic variability in these objects (see Section 3 for GUFI specs). Throughout this campaign, we have consistently achieved photometric precisions of $<0.5 \%$ (and as low as $0.15 \%$ for some observations) as shown in Table 3, as well as sampling many rotation periods per object, per night. By contrast, the above studies have typically achieved photometric precisions of $\geqslant 1.0 \%$ (with some as low as $\sim 0.5 \%$ ), and in many cases, the rotational phase has been poorly covered. Furthermore, large-survey data sets only produced a few data points per hour in order to contemporaneously monitor a large number of objects. Thus, a combination of high photometric precision, well sampled rotational phase coverage, and high cadence data sets are perhaps crucial in 

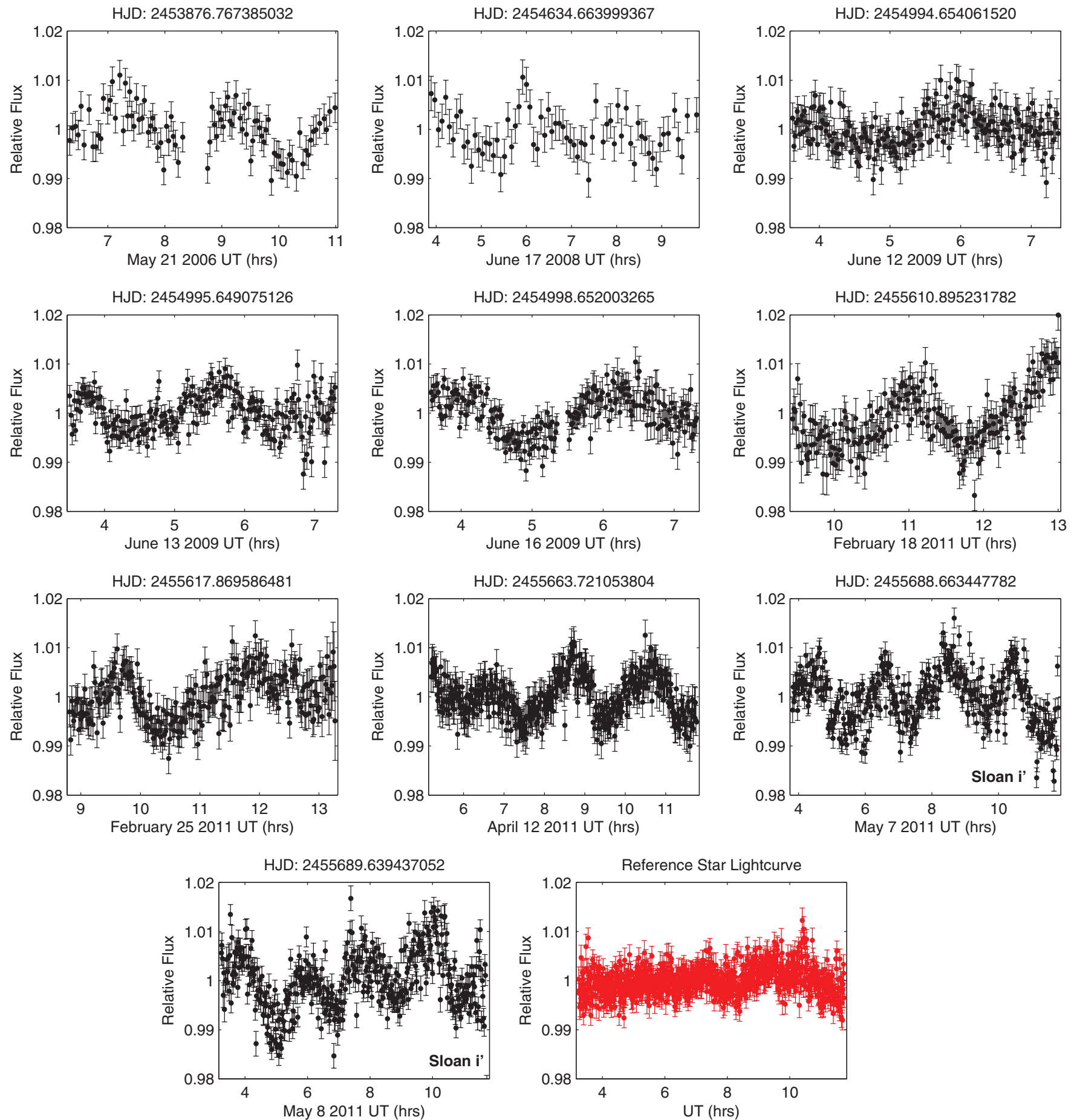

Figure 5. TVLM 513-46546: we obtained $\sim 53 \mathrm{hr}$ of data, over a $\sim 5 \mathrm{yr}$ baseline for TVLM 513 . Our data shows an extremely stable period of $1.95958 \pm 0.00005 \mathrm{hr}$, which we phase connect over this baseline. The data shown here was taken in the $I$ band $(\sim 7000-11,000 \AA)$ and Sloan $i^{\prime}(\sim 6500-9500 \AA)$, which is marked on the relevant light curves (2011 May 7 and 8). This confirmed period further constrains the work of Lane et al. (2007) who found a photometric period of $\sim 1.96$ hr, also in the $I$ band. As in the case of LSR J1835, this periodicity is consistent with the observations of Hallinan et al. (2006, 2007), who report periodic radio pulses of $\sim 1.96 \mathrm{hr}$ for TVLM 513. In this work, we investigate the stability of the light curve phase and amplitude, and find the phase to be stable throughout each data set, where changes in amplitude are present $\left(0.56 \%-1.20 \%\right.$ in the $I$ band and $0.92 \%-0.96 \%$ in Sloan $\left.i^{\prime}\right)$. We discuss this further in the following section. As always, a randomly selected reference star light curve is included (bottom right).

(A color version of this figure is available in the online journal.)

effectively detecting periodic variability from ultracool dwarfs on these timescales. In particular, we highlight that two dwarfs from our sample, 2M J0746AB and 2M J0036, were included in surveys mentioned above (Clarke et al. 2002a; Maiti 2007). In both cases, variability was detected, albeit with insufficient phase coverage to recover the periodic signal detected in our work.

However, we cite Bailer-Jones \& Mundt (2001) and Rockenfeller et al. (2006a) as reliable comparison studies (with similar sensitivities to the periodic variability reported 

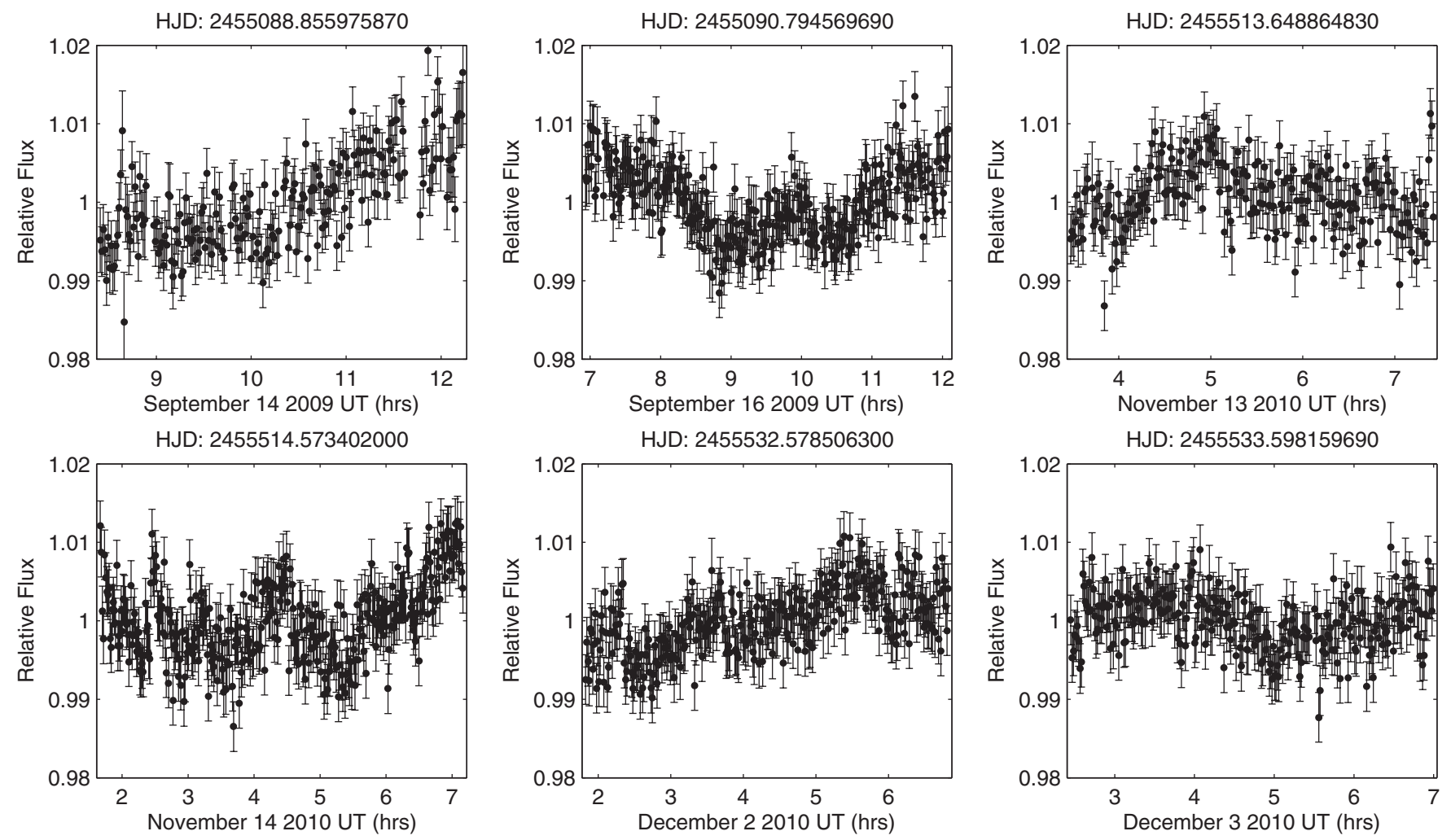

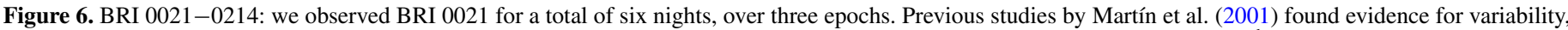

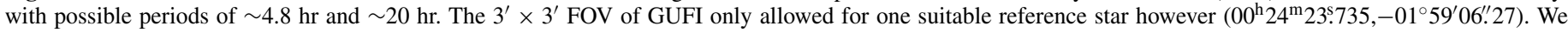

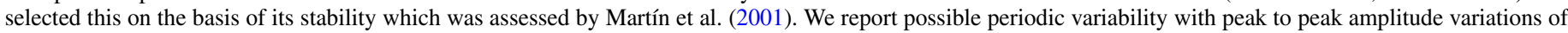

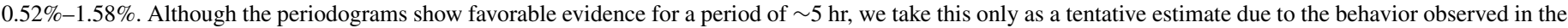

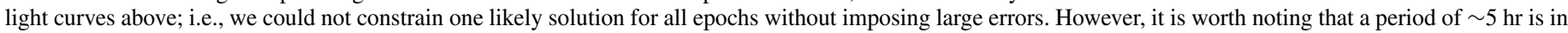

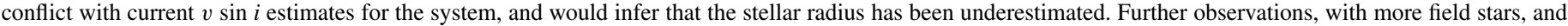
larger temporal coverage are needed to effectively assess the photometric behavior of this object.

in this work) that searched for variability (including periodic variability) in a sample of dwarfs that were not pre-selected as radio-detected. We select objects in these papers between $\geqslant$ M7.5-L3.5 only, in order to satisfy a direct comparison to the objects in this work. In the case of Bailer-Jones \& Mundt (2001), they observed $21 \mathrm{M}$ and L type dwarfs, where 15 of these occupy the spectral range we define above. Similarly, Rockenfeller et al. (2006a) cover a sample of $19 \mathrm{M}$ dwarfs, where 6 of these are M7.5-M9. Crucially, their work provided detection limits of $\sim 0.5 \%-5 \%$ in the PtP amplitude variations of variable targets. In addition to this, the methods of Bailer-Jones \& Mundt (2001) were sensitive to periods $>1 \mathrm{hr}$, and Rockenfeller et al. (2006a) for $\sim 0.5-12 \mathrm{hr}$. Therefore, based on the PtP amplitudes detected in our work, their studies both had the capability of detecting the presence of periodic variability in their sample. However, although Bailer-Jones \& Mundt (2001) report evidence of some periodic signals, they only report tentative detections from some targets. Similarly, Rockenfeller et al. (2006a) detect periodic optical variability from only one of six sources-the M9 dwarf 2MASSW J1707183+643933. During these observations, they also report a large flare event (Rockenfeller et al. 2006b), and as a result, argue that the presence of magnetic activity is expected. Gizis et al. (2000) reported $\mathrm{H} \alpha$ emission for the same M9 dwarf with an equivalent width of $9.8 \AA$, further supporting the possible presence of magnetic activity.

The presence of consistent periodic variability in five of six radio-detected ultracool dwarfs demonstrates that the correlation between optical and radio periodic variability is significant, and thus, the presence of magnetic activity is also significant when compared to the above studies. Therefore, we have a case to highlight an expected presence of consistent periodic optical variability in radio-detected sources, due to the presence of strong magnetic fields $(\mathrm{kG})$ with radio activity. We note further that all of our target sample consists of rapid rotators, with high $v \sin i$ values $\left(>15 \mathrm{~km} \mathrm{~s}^{-1}\right)$. This is perhaps an additional bias in our data, whereby rapid rotators could be more easily detected than slowly rotating sources. However, an expanded sample of non-radio active dwarfs that are also rapid rotators, is required to quantify this further.

Previous studies have argued that magnetic spots (e.g., Rockenfeller et al. 2006a; Lane et al. 2007) or dust (e.g., BailerJones \& Mundt 2001; Littlefair et al. 2008) were responsible for similar detected periodicities in ultracool dwarfs. One possible means of distinguishing between various mechanisms is to compare simultaneous multi-band photometry to synthetic atmospheric models (Allard et al. 2001). Importantly, in order to carry out such analyses, simultaneous observations are needed due to the inherent variability in the amplitude of the optical variability. The studies of Rockenfeller et al. (2006a) and Littlefair et al. (2008), for example, yielded cases in favor of both cool magnetic spots and the presence of atmospheric dust, respectively. These results were based on the ratios of the PtP amplitude variations at different photometric wavebands. Despite detecting larger PtP amplitude variations in $R$-band versus $I$-band for two of our target sample (see Table 3), we did not obtain simultaneous photometry and therefore cannot apply these models at this point. However, such a high detection rate of significant periodic variability in our sample of 

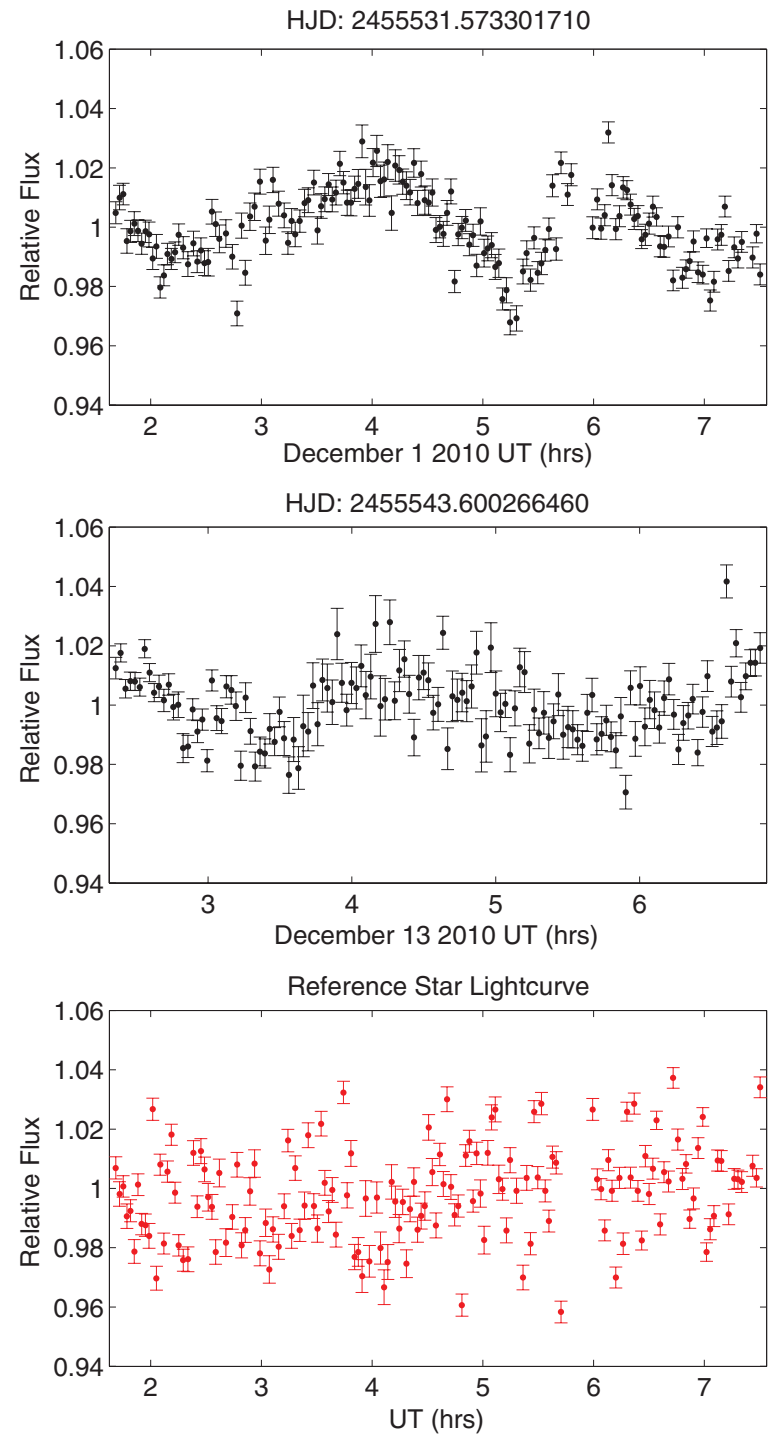

Figure 7. 2MASS J0036+18: we confirm a period of $3.0 \pm 0.7 \mathrm{hr}$ for $2 \mathrm{M}$ J0036. Unfortunately, both nights of observation were subject to poor weather conditions (heavy cloud). Nevertheless, our range of periods are in agreement with the observations of Lane et al. (2007), who detect a $\sim 3 \mathrm{hr}$ period for this source in the Johnson $I$ band. Berger et al. (2005) and Hallinan et al. (2008) showed this dwarf to be radio pulsing with a period of $3.08 \pm 0.05 \mathrm{hr}$. We note that the light curves above were binned to two-minute frames in order to increase the $\mathrm{S} / \mathrm{N}$.

(A color version of this figure is available in the online journal.)

radioactive dwarfs, implies a correlation with radio activity and thus some kind of magnetic phenomenon. The nature of this optical variability will be addressed in an upcoming paper focused on spectro photometric observations of such targets (G. Hallinan et al., in preparation).

\subsection{The Phase Stability of TVLM 513-46546}

A number of ultracool dwarfs have shown periodic behavior over a number of observations (e.g., Berger et al. 2005; Hallinan et al. 2006, 2007, 2008; Lane et al. 2007; Littlefair et al. 2008); here, we use multi-epoch observations of one dwarf, TVLM 513, to investigate whether this periodicity is long term and stable in phase and whether this modulation evolves morphologically over these timescales. We achieve an accurate enough period of rotation of $1.95958 \mathrm{hr}$ for the dwarf via phase connection of the 2006-2011 epochs, with an associated error in the period of
$0.00005 \mathrm{hr}$, thereby allowing us to assess its modulated behavior over the $\sim 5$ yr campaign. We find long-term, periodic variability that is stable in phase as shown in Figure 9, where we overplot a model sinusoidal signal (red) over the entire baseline. Phase folded light curves of individual observations are shown in Plot 1 of Figure 9, once again highlighting this agreement, and similarly in Plots 6 and 7 we show phase folded light curves of all datasets.

Such a high degree of correlation suggests a spatially stable surface feature that does not appear to move by a significant amount over this baseline. Donati et al. (2006) and Morin et al. (2010) have shown that large-scale magnetic fields for fully convective objects are stable on year-long timescales. Hallinan et al. (2006, 2007, 2008) confirmed the presence of stable kG magnetic fields for TVLM 513, consistent with a common magnetic field-related origin for the periodic radio and optical variability for ultracool dwarfs, as discussed in the previous section.

While stable in phase, the PtP amplitude is variable during this campaign from $\sim 0.56 \%$ to $1.20 \%$ in the VATT $I$ band (see Table 3). Since the phase is stable, a change in amplitude suggests that the intensity of the feature responsible is changing on these levels, or that it may be changing in size. Littlefair et al. (2008) observed PtP variations in Sloan $i^{\prime}$ of $0.15 \%$. Here, we report much larger Sloan $i^{\prime} \mathrm{PtP}$ variability of $0.92 \%-0.96 \%$ - further evidence of variable PtP amplitudes when compared to other studies. This is intriguing when compared to the previous radio activity discussed by Hallinan et al. (2006, 2007), who reported highly variable signals from TVLM 513. Specifically, they detected bursts of periodic radio emission that varied greatly between epochs, also indicating changes in emission intensities. Whether the optical emission here is directly related to radio variability will be conclusively determined when multiple epochs of radio data are obtained, and phase connected, over the same timescales as this work.

\subsection{The Radio and Optical Emission at Odds from 2MASS J0746425+200032AB?}

In this work, we have demonstrated evidence of a correlation between the optical and radio variabilities in ultracool dwarfs. Therefore, we briefly consider why we detect optical periodic variability from the non-radio-detected binary component of the 2M J0746AB system.

According to model-derived temperature estimates of Konopacky et al. (2010), the effective temperature of $2 \mathrm{M}$ J0746A $\left(T_{\text {eff }} \sim 2205 \pm 50 \mathrm{~K}\right)$ is higher than its counterpart $\left(T_{\text {eff }} \sim 2060 \pm 70 \mathrm{~K}\right)$. As previously discussed, our photometry contains the combined flux of both stars-perhaps the contrast ratios of stellar photosphere versus feature are much greater for $2 \mathrm{M} \mathrm{J} 0746 \mathrm{~A}$ as a result. If the optical and radio emission are linked as we put forward as a possibility, why did Berger et al. (2009) not also observe some evidence of radio emission from 2M J0746A?

The primary could be pulsing at radio frequencies, but undetectable due to the inclination angle of the system. However, Harding et al. (2013) find that the 2M J0746AB rotation axes are orthogonally aligned to the system orbital plane. This established alignment geometry could support detectable beaming from both stars. However, this is contingent upon the magnetic field alignment of each star being equal with respect to their rotation axes. Misaligned magnetic field axes could mean that the radio emission from $2 \mathrm{M} \mathrm{J0746A}$ is being beamed away from the observer. Alternatively, unlike $2 \mathrm{M} \mathrm{J} 0746 \mathrm{~B}$, it is 

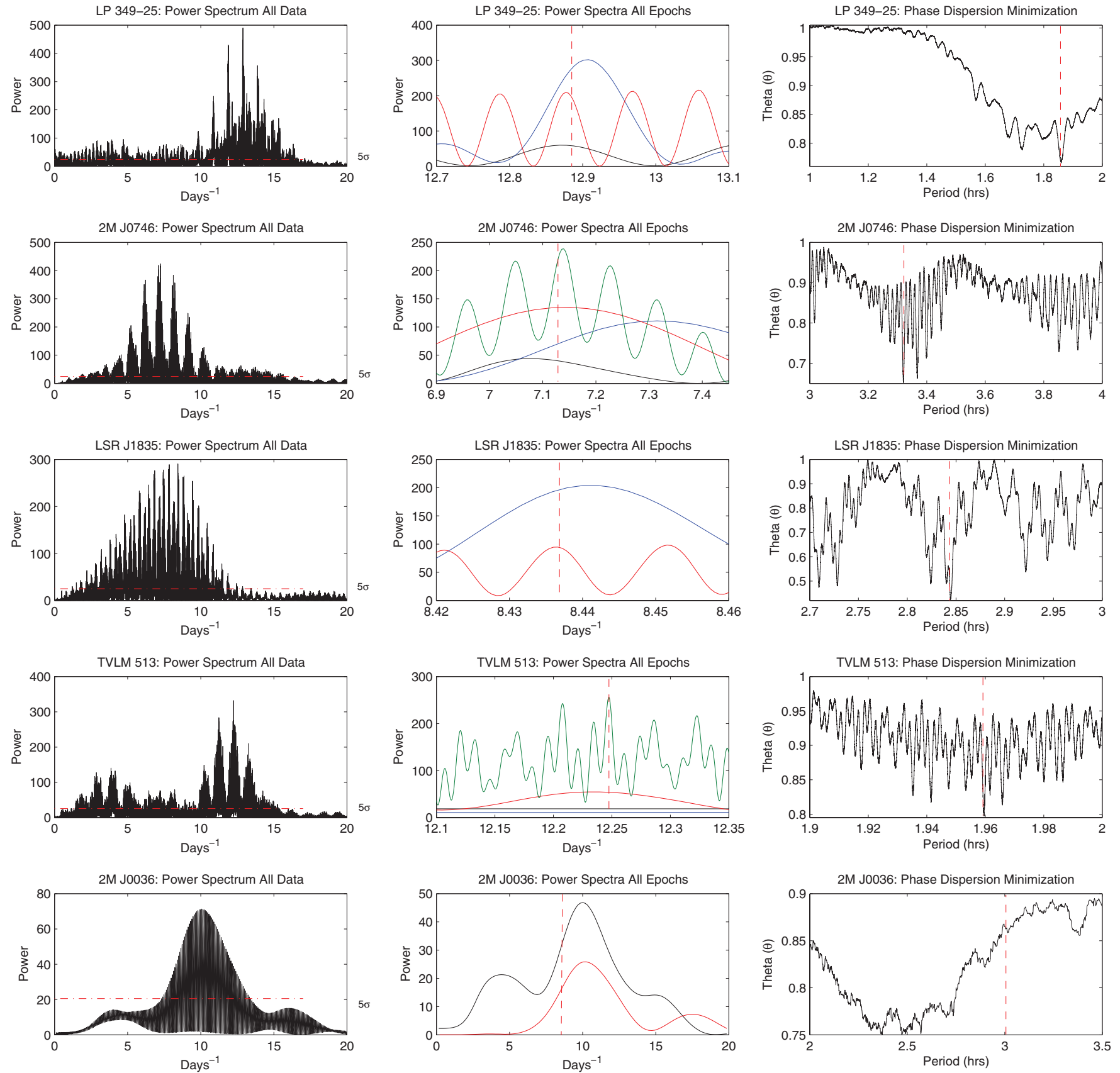

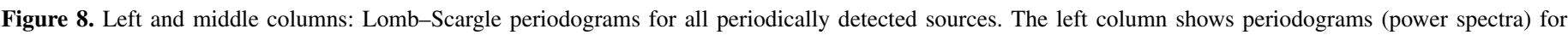

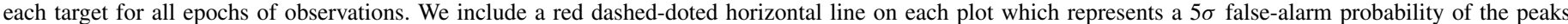

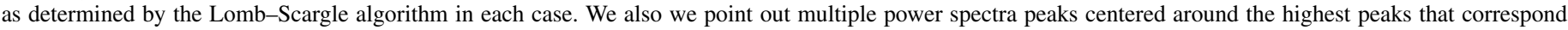

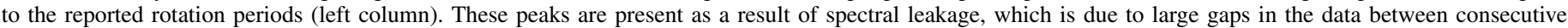

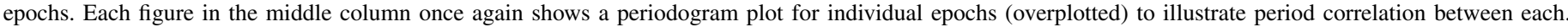

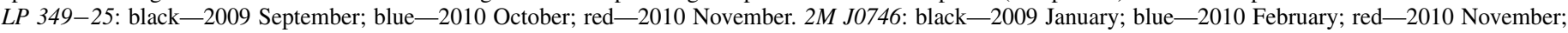

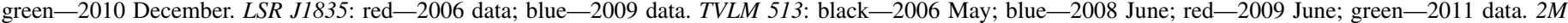

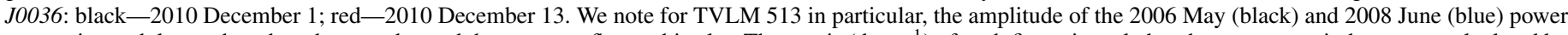

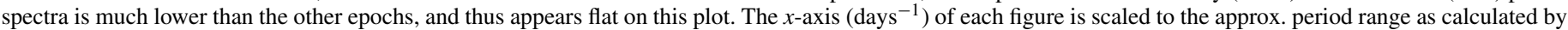

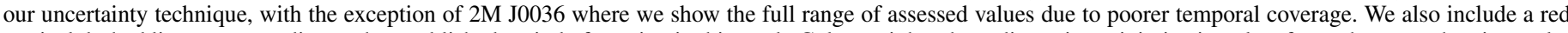

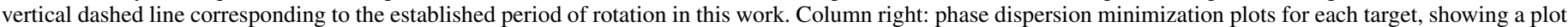

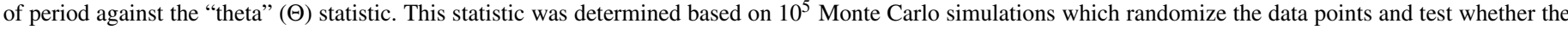

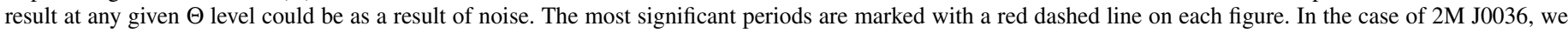

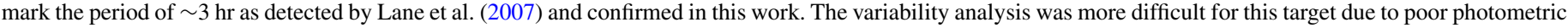
conditions.

(A color version of this figure is available in the online journal.)

also possible that 2M J0746A does not exhibit beamed ECM emission at all, but perhaps only small levels of quiescent radio emission that has not yet been detected by previous studies of the system. Speculating further about the intricacies of the system's radio emission and the associated beaming geometry is outside the scope of this work.

Some aperiodic variability is also present for some observations which could be due to the contribution from a weaker 

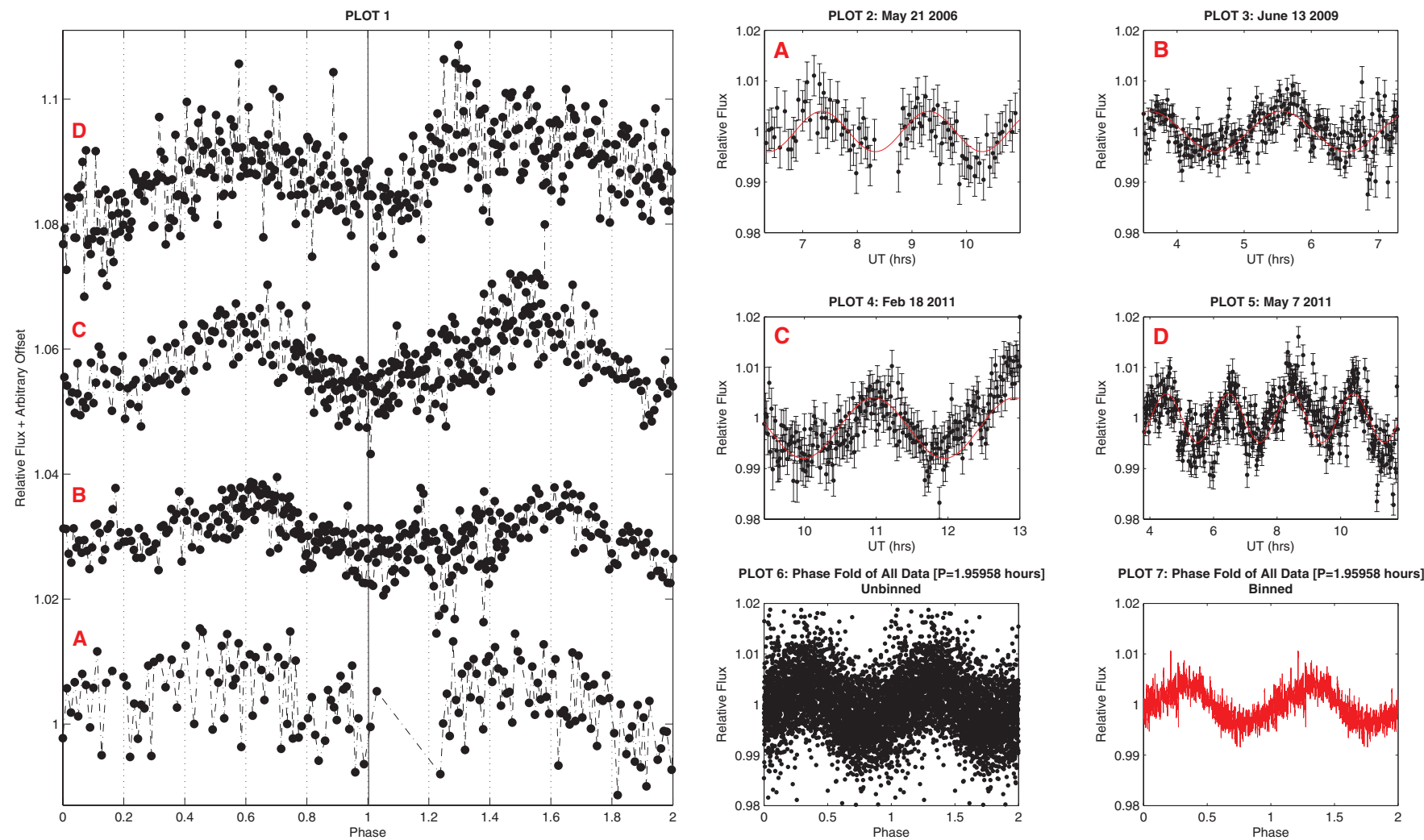

Figure 9. Plot 1: this figure illustrates the phase stability of the periodic variability of TVLM 513 over a $\sim 5$ yr baseline. These raw light curves, labeled with red letters A-D (bottom-top), were selected at random from four of the observation epochs (2006 May-2011 May). This level of agreement is consistent for all light curves in the sample. In each case, the time stamps were phase folded to the period of $1.95958 \mathrm{hr}$. Plots 2-5: to show this agreement further, the light curves A, B, C, and D in Plot 1 correspond to Plots 2-5, respectively. Each light curve contains an overplotted model sinusoidal signal (red), with a period of $1.95958 \mathrm{hr}$, which was applied to the full 2006-2011 dataset, where we set values between individual observations and epochs to zero. It is clear that this dwarf exhibits highly correlated behavior in terms of phase over this baseline, and furthermore, that the stellar feature responsible must be equally as stable (spatially) during these observations. Plot 6 and Plot 7 : we phase fold the entire data set (2006-2011, containing 3500 data points) to the detected period of 1.95958 hr. The black phase folded light curve in Plot 6 is raw and has no binning or scaling. The red phase folded light curve in Plot 7, once again of all data, has been binned by a factor of 10.

(A color version of this figure is available in the online journal.)

secondary signal. The LS periodogram analysis in this work should extract both photometric signals if they are both present and strong enough, and our data shows strong evidence of variability of the non-radio emitting component. Resolved photometry would be an interesting confirmation if the radio active source is also optically variable.

\subsection{The Unusual Behavior of LP 349-25}

In this section, we discuss the behavior of the light curves of the binary LP 349-25AB. As outlined in Section 5.2, we observe significant changes in amplitude in the $I$ band (refer to Table 3), as well as changes in phase during single observations. Due to the close separation of the binary members, the photometric aperture used enclosed the combined flux of both components. Therefore, the presence of two periodically varying sources in these data, and thus the superposition of these waves, is one possible explanation for the varying amplitude we observe here. However, aperiodic variability of a single periodic source could also cause this behavior. This is an obvious distinction and one that we discuss below.

We first consider the possibility of the presence of two periodically varying sources by subtracting the main $1.86 \mathrm{hr}$ period out of the raw data. We did this by generating a sinusoidal model wave function with a period of $1.86 \mathrm{hr}$. We then iterated through a range of amplitude and phase values, and performed a LSF to the raw data from the 2010 October epoch. We chose this set of data because we had contiguous observation nights from 2010 October 9-15 UT, as shown in Figure 10. The best solution which fitted the raw data parameters was subtracted out. LS periodogram analysis was run on the remaining data points, which searched for residual periodic signatures. We observed no obvious evidence in the periodogram of any second significant source. As a follow-up, we modeled the superposition of two sinusoidal sources by setting a period of $1.86 \mathrm{hr}$ for one source, varying the other period, as well as the amplitude and the phase of both waves, and performed a LSF to our data-as outlined in Section 4.3. These fits did not yield strong evidence of another source based on the best LSF solutions. The lack of evidence in the periodograms, as well as the inability to clearly detect an underlying source in the residual data after subtracting the main $1.86 \mathrm{hr}$ period out, does not support the obvious presence of another period.

Nevertheless, the varying component of amplitude and phase remains in these data, as shown in Figure 10. In this plot, we show raw light curves from the 2010 October epoch (October 10-15 UT) with a model sinusoidal wave overplotted (in red). The established period of $1.86 \mathrm{hr}$ was used, and corresponding amplitudes from Table 3 were adopted for each light curve. We use a fixed phase for all nights. As we observe the model wave for each observation, we can see that the wave is in phase for some nights (e.g., October 11, 13 and 15). By contrast, the signal appears to have moved out of phase for October 12. We can also see, for October 10 and 14, for example, that the model is largely 

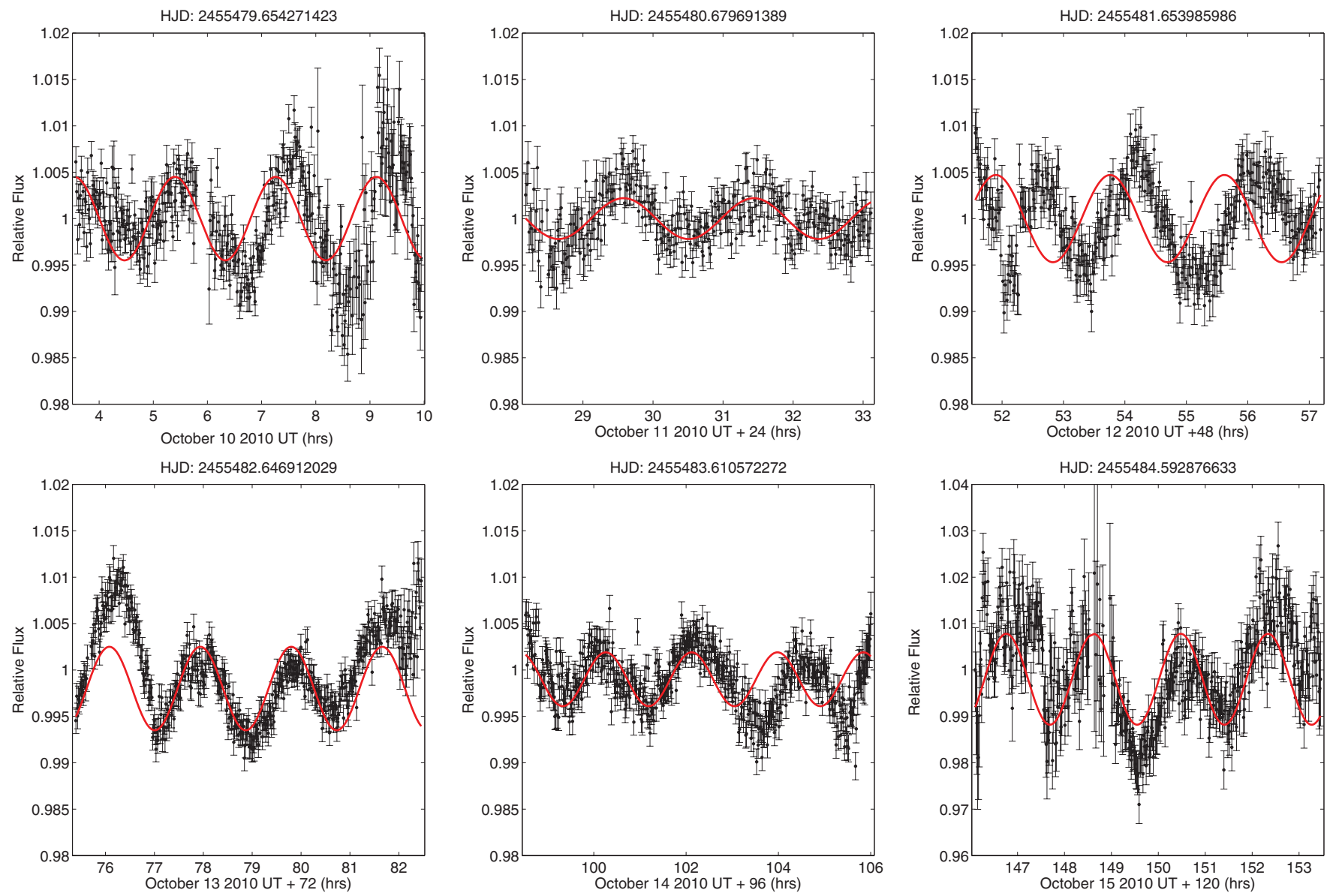

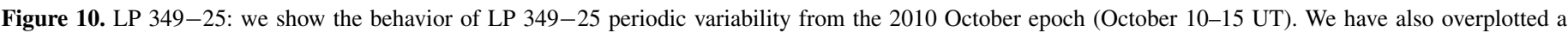

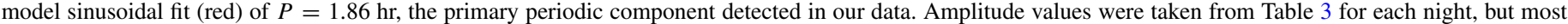

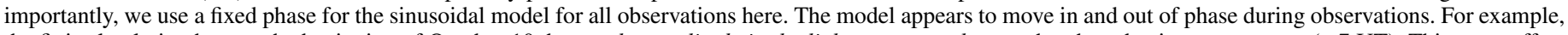

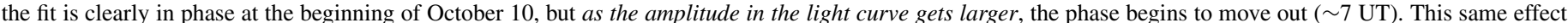

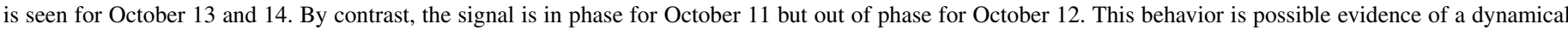

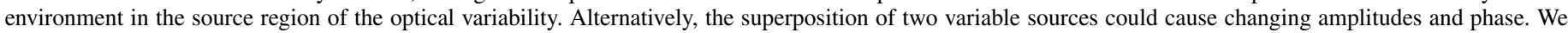
cite TVLM 513 (Figure 9) as an example of a source exhibiting consistent phase stability for an established period.

(A color version of this figure is available in the online journal.)

in phase for the first half of each observation (although upon closer inspection there is some evidence of trailing and leading peaks and troughs), but then moves partially out of phase as the amplitude of the signal increases-we also note changes in light curve morphology for these sections.

This behavior could be characteristic of a high-dynamic environment in these regions, where the source of the variability is evolving on these timescales. Perhaps a magnetic feature is not stationary on the stellar photosphere, or alternatively, a combination of features could be affecting light curve morphology. Moreover, if these features were undergoing changes in size or temperature, this could also have an effect on the sinusoidal shape. We can not rule out the possibility of another source-perhaps a more robust modeling technique than those used here is required to identify the presence of another period. Obtaining a contiguous time series of LP 349-25 over many periods of rotation, would allow us to more effectively investigate whether these morphological changes are evolving in a systematic and repeatable manner.

\subsection{Spin-Orbit Alignment of LP 349-25AB}

The detected rotation period from LP 349-25B in this work provides an important parameter in assessing the orbital copla- narity of the system, as well as the associated implications for binary formation theory in the VLM binary regime. Recent work by Harding et al. (2013) demonstrated spin-orbit alignment for the VLM binary 2M J0746AB - the first such observational result in this mass range. Their work showed that the spin axes inclinations of both components of the system were aligned to within 10 degrees of the orbital plane. Such an alignment signals that solar-type binary formation mechanisms, such as core fragmentation, disk fragmentation, or competitive accretion, may extend into the realm of brown dwarfs. Although the alignment of one system could not be used to distinguish between the various formation theories, investigating such alignments in other VLM systems provides an insight into where the above formation pathways may dominate. Here, we applied the same approach as outlined in Harding et al. (2013) to assess the orbital properties LP 349-25AB.

\subsubsection{Estimating Age and Mass}

We used the evolutionary models of Chabrier et al. (2000) to estimate the age and mass (and later the radius) of each binary component. These parameters were constrained by adopting the established total system mass of $0.121 \pm 0.009 M_{\odot}$, as well as the photometric $J H K$ measurements and bolometric luminosity 


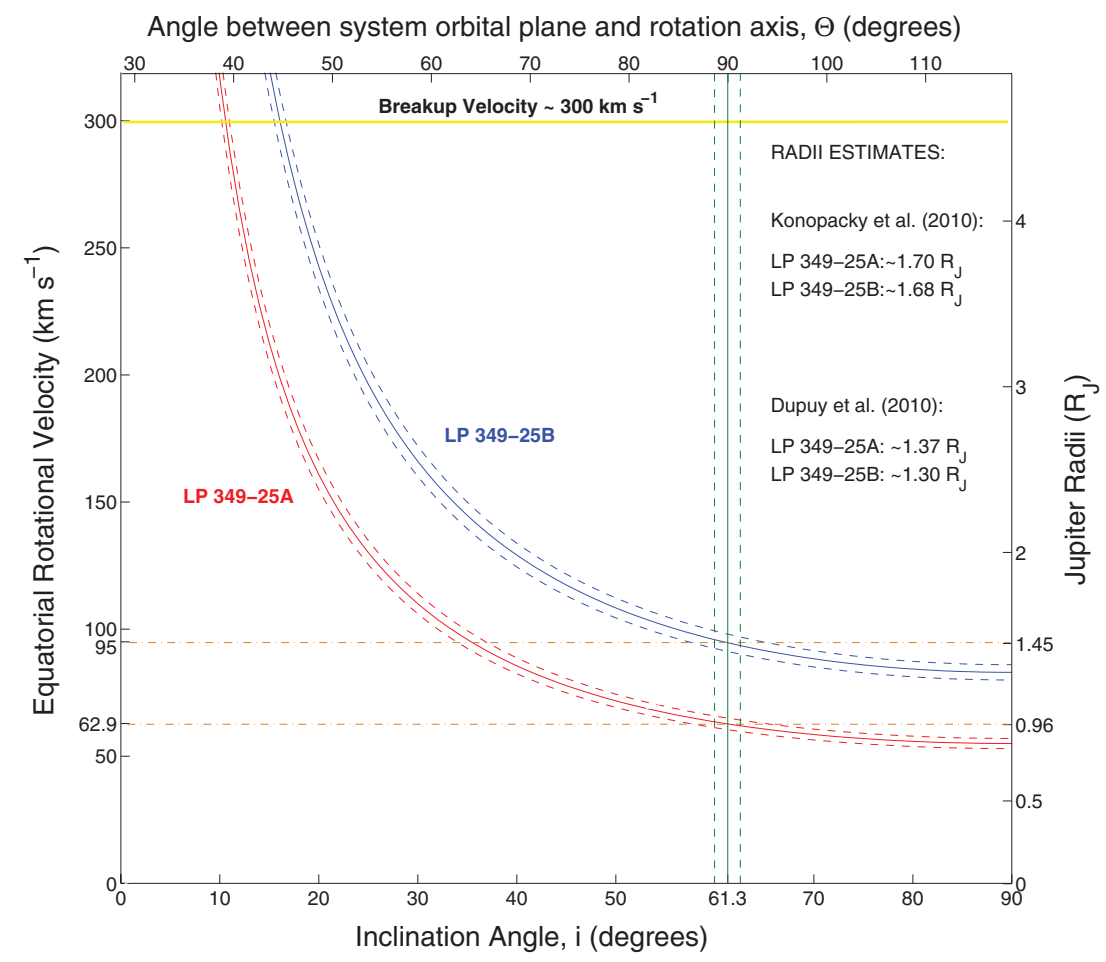

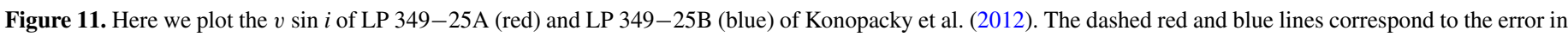

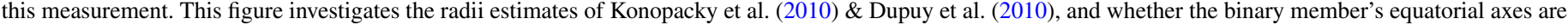

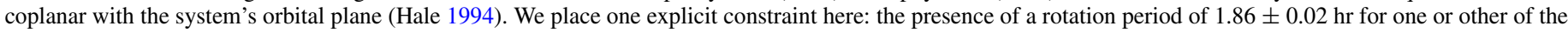

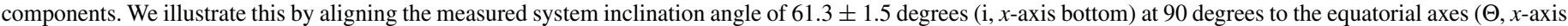

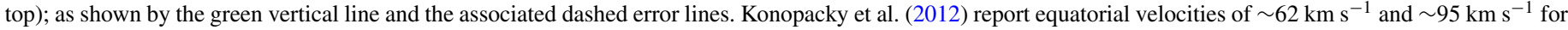

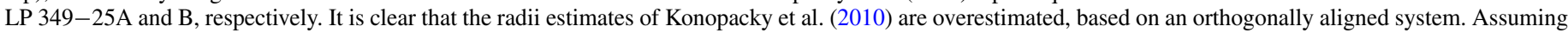

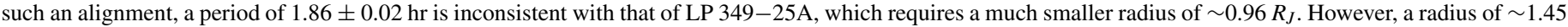

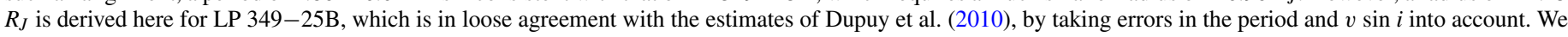

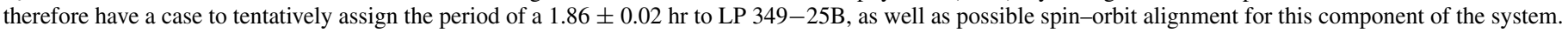

(A color version of this figure is available in the online journal.)

measurements of Konopacky et al. (2010). In addition, previous spectroscopic investigations yielded no lithium in the dwarf's spectrum, e.g., Bouy et al. (2004). We used these parameters to identify a range of ages where lithium was absent, and next, interpolated over a range of masses by comparing the correlation between the $J H K$ colors and bolometric luminosities of Konopacky et al. (2010) and those of the Chabrier et al. (2000) models. Furthermore, by assuming each component was coeval, the sum of the component masses could not exceed the measured total system mass of $0.121 \pm 0.009 M_{\odot}$.

We find an age consistent with Dupuy et al. (2010) of $\sim 140 \mathrm{Myr}$, with masses of $\sim 0.06 M_{\odot}$ and $\sim 0.05 M_{\odot}$ for LP 349-25A and LP 349-25B, respectively. However, lithium is present in this range. Dupuy et al. (2010) suggested that perhaps the absence of lithium in the binary spectrum was due to flux domination from the primary member, and given the predicted mass of LP 349-25B in their work, the $\mathrm{Li}_{I}$ doublet is expected since LP 349-25B potentially lies below the theoretically predicted lithium depletion point at $\approx 0.055-0.065 M_{\odot}$. The only ages (where $\mathrm{Li}=0$ ) that are in mild agreement suggest that the system has a total mass that far exceeds $0.121 \pm 0.009 M_{\odot}$. Lithium, however, may not be a robust indicator of age. For example, Baraffe \& Chabrier (2010) point out that episodic accretion can cause lithium to be depleted at younger ages, despite its expected presence based on evolutionary models. Another possibility might also be that the total system mass has been under estimated, which would place LP $349-25 \mathrm{AB}$ at an older age in the models of Chabrier et al. (2000), consequently supporting the observed absence of lithium.

\subsubsection{Radius and Inferred Spin-Orbit Alignment}

Dupuy et al. (2010) obtained dynamical mass measurements of a sample of late-M dwarfs, including LP 349-25AB. Their modeling subsequently yields radii estimates of $\sim 1.30-1.44 R_{J}$ for LP 349-25A and $\sim 1.24-1.37 R_{J}$ for LP $349-25 \mathrm{~B}$. However, Konopacky et al. (2010) find much larger radii estimates of $1.7_{-0.09}^{+0.08} R_{J}(\mathrm{~A})$ and $1.68_{-0.08}^{+0.09} R_{J}(\mathrm{~B})$. These studies based their radii on evolutionary model-derived parameters (Burrows et al. 1997; Chabrier et al. 2000; Allard et al. 2001). Under the assumption of a perfectly coplanar spin-orbit alignment, by adopting the individual rotational velocity measurements of Konopacky et al. (2012) and by assigning the detected period in this work of $1.86 \pm 0.02 \mathrm{hr}$ to each component, we derive radii of $\sim 0.96 R_{J}$ for LP $349-25 \mathrm{~A}$ and $\sim 1.45 R_{J}$ for LP $349-25 \mathrm{~B}$. We show these in Figure 11 by the dash-dotted horizontal lines, where we have plotted the system's equatorial velocity versus inclination angle (refer to caption).

Considering the radii estimates of Konopacky et al. (2010), as well as an orbital inclination angle of $61.3 \pm 1.5$ degrees from their work, we derive a maximum period of rotation of $\sim 3.77 \mathrm{hr}$ and $\sim 2.47 \mathrm{hr}$ for LP $349-25 \mathrm{~A}$ and LP $349-25 \mathrm{~B}$, respectively. Indeed, these radii estimates appear to be very large when considering the evolutionary models of Chabrier et al. (2000) for a given range of ages, $L_{\mathrm{bol}}$, and total system mass presented in their work, in addition to a lack of detected lithium in the binary spectra (Reiners \& Basri 2009). Therefore, it is difficult to infer which component matches our detected period. 
Orbital Coplanarity of LP349-25AB?

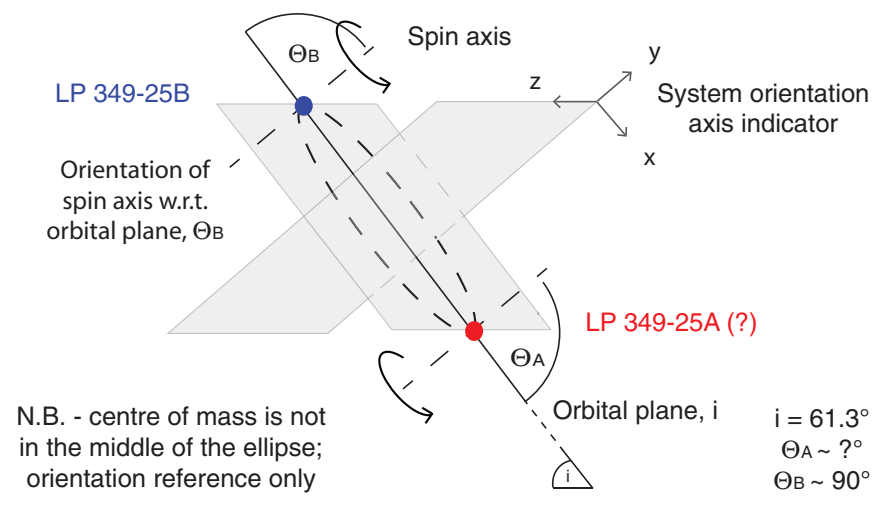

Figure 12. Sketch of the configuration of LP 349-25AB, which loosely illustrates the possible system orientation. Based on a radius estimate for LP 349-25B of $\sim 1.37 R_{J}$ (Dupuy et al. 2010), in addition to the $v \sin i$ of $83 \pm 3 \mathrm{~km} \mathrm{~s}^{-1}$ (Konopacky et al. 2012), and the period of $1.86 \pm 0.2 \mathrm{hr}$ in this work, there is tentative evidence that the orientation of the equatorial axis of LP $349-25 \mathrm{~B}, \Theta_{B}$, is perpendicularly aligned with the binary orbital plane.

(A color version of this figure is available in the online journal.)

As previously noted, the Dupuy et al. (2010) binary radii instead infer maximum periods of $\sim 2.65 \mathrm{hr}$ and $\sim 1.67 \mathrm{hr}$ respectively. This discrepancy could be due to the fact that Konopacky et al. (2010) use only broadband photometry, and furthermore, use the effective temperature as one of the inputs for model-predicted mass, whereas Dupuy et al. (2010) obtain their temperature estimates via NIR fitting, which is $\sim 650 \mathrm{~K}$ higher. Notably, determining an accurate estimate of the radius of young, magnetically active stars can be very difficult based on the effect of a reduction in convective efficiency of such objects $\left(<0.35 M_{\odot}\right.$; see Chabrier et al. 2007). Since the adiabatic properties of a star increase with mass, such an environment reduces convection in the outer areas. The end result is a reduction in stellar luminosity and core temperature, causing the radius to expand.

Nevertheless, a radius estimate of $\sim 1.45 R_{J}$ for LP $349-25 \mathrm{~B}$ (derived above) is in loose agreement with the estimates of Dupuy et al. (2010), and therefore, we highlight a tentative spin-orbit alignment for the secondary star-as shown by the sketch in Figure 12. Establishing the period of rotation of the other binary component will enable a more effective constraint of the orbital properties. Finally, as in the case of 2M J0746AB, the inclinations of the spin axes with respect to our line of sight may be equal, but this does not always imply that the orbital planes are perpendicularly aligned. Even if edge-on systems are orthogonal to the sky, they could be coincidentally equal. We refer the reader to Harding et al. (2013) for a discussion of the various formation mechanisms and the implications for formation theory in the VLM binary regime.

\section{SUMMARY AND CONCLUSIONS}

We have reported on optical photometric observations of six ultracool dwarfs spanning the $\sim$ M8-L3.5 spectral range. Our work has confirmed periodic optical variability for five out of six of the radio active dwarf samples, where periodicity for two of these was discovered for the first time. We report a tentative detection of periodic variability for another dwarf-sampling the rotational phase of this object will establish whether periodic variability is also present. Based on previous surveys that have yielded a low fraction of periodic variability for late-M and $\mathrm{L}$ dwarfs, our results indicate a likely correlation between the optical and radio periodic variability. This correlation implies that the optical and radio periodic emissions may be related by some kind of magnetic phenomena; however, at this point, it is not clear whether such a possible connection is causal in nature.

For one of our targets in particular, the pulsing M9 ultracool dwarf TVLM 513, we find periodic variability that is extremely stable in phase over baselines of $\sim 5 \mathrm{yr}$. We achieved an accurate enough rotation period of $1.95958 \pm 0.00005 \mathrm{hr}$ that allowed us to phase connect the $\sim 5 \mathrm{yr}$ baseline. The high level of phase stability indicates that the stellar feature responsible for the periodic variability is not moving over these timescales. We do however observe large changes in the PtP amplitude variability, pointing toward changes in the size or intensity of the source regions responsible for the periodicity.

Similarly, for the M tight binary dwarf LP 349-25, the PtP amplitude variations change significantly during observations. The phase also changes on these timescales, where we observe it to move in and out of phase during single nights. A number of scenarios are considered for this behavior-e.g., the presence of two periodically varying sources. These changes in morphology could also be a consequence of a high-dynamic environment in these regions, where features are changing in size, temperature, and shape, and/or are moving with respect to the stellar photosphere.

Finally, we assess the spin-orbit alignment of LP 349-25, based on the discovery of the rotation period for one component in this work. By adopting the radii estimates of Dupuy et al. (2010), and assigning the period of rotation of $1.86 \mathrm{hr}$ discovered here for LP 349-25B, we find evidence for a tentative alignment of the spin-orbital axes. Such an alignment has been observed for another VLM binary dwarf-2M J0746AB (Harding et al. 2013). Establishing the second period of rotation for the system would further constrain its orbital properties and provide further insight into the possible formation mechanisms responsible for such alignments in the VLM binary regime.

This work was largely carried out under the National University of Ireland Traveling Studentship in the Sciences (Physics). L.K.H. gratefully acknowledges the support of the Science Foundation Ireland (Grant Number 07/RFP/PHYF553). We thank the VATT team for their help and guidance, especially Dave Harvey, Michael Franz, and Ken Duffek. L.K.H. personally thanks Dr. Mark Lang for his constant assistance, in addition to the staff of the NRAO who provided significant support when L.K.H. was conducting some of his work during his appointment as an NRAO graduate intern. We extend our thanks to Dr. Stuart Littlefair for his many helpful comments on this work. Finally, we thank the editor, Dr. Steven Kawaler, in addition to the referee for their careful reading of our work and for their helpful input on how to improve this manuscript.

\section{REFERENCES}

Akerlof, C., Alcock, C., Allsman, R., et al. 1994, ApJ, 436, 787

Allard, F., Hauschildt, P. H., Alexander, D. R., Tamanai, A., \& Schweitzer, A. 2001, ApJ, 556, 357

Antonova, A., Doyle, J. G., Hallinan, G., Bourke, S., \& Golden, A. 2008, ApJ, 487,317

Antonova, A., Doyle, J. G., Hallinan, G., Golden, A., \& Koen, C. 2007, A\&A, 472, 257

Artigau, É., Bouchard, S., Doyon, R., \& Lafrenière, D. 2009, ApJ, 701, 1534

Bailer-Jones, C. A. L. 2004, A\&A, 419, 703

Bailer-Jones, C. A. L., \& Mundt, R. 1999, A\&A, 348, 800

Bailer-Jones, C. A. L., \& Mundt, R. 2001, ApJ, 367, 218 
Baraffe, I., \& Chabrier, G. 2010, A\&A, 521, 44

Basri, G. 2001, in ASP Conf. Proc. 223, Proceedings of the 11th Cambridge Workshop on Cool Stars, Stellar Systems and the Sun, ed. R. J. Garcia Lopez,

R. Rebolo, \& M. R. Z. Osorio (San Francisco, CA: ASP), 261

Basri, G., \& Marcy, W. M. 1995, AJ, 109, 762B

Benz, A. O., \& Güdel, M. 1994, A\&A, 285, 621

Berger, E. 2002, ApJ, 572, 503

Berger, E. 2006, ApJ, 648, 629

Berger, E., Ball, S., Becker, K. M., et al. 2001, Natur, 410, 338

Berger, E., Basri, G., Fleming, T. A., et al. 2010, ApJ, 709, 332

Berger, E., Basri, G., Gizis, J. E., et al. 2008a, ApJ, 676, 1307

Berger, E., Gizis, J. E., Giampapa, M. S., et al. 2008b, ApJ, 673, 1080

Berger, E., Rutledge, R. E., Phan-Bao, N., et al. 2009, ApJ, 695, 310

Berger, E., Rutledge, R. E., Reid, I. N., et al. 2005, ApJ, 627, 960

Bouy, H., Duchêne, G., Köhler, R., et al. 2004, A\&A, 423, 341

Burgasser, A. J., \& Putman, M. E. 2005, ApJ, 626, 486

Burrows, A., Marley, M., Hubbard, W. B., et al. 1997, ApJ, 491, 856

Chabrier, G., Baraffe, I., Allard, F., \& Hauschildt, P. 2000, ApJ, 542, 464

Chabrier, G., Gallardo, J., \& Baraffe, I. 2007, A\&A, 472, L17

Clarke, F. J., Hodgkin, S. T., Oppenheimer, B. R., Robertson, J., \& Haubois, X. 2008, MNRAS, 386, 2009

Clarke, F. J., Oppenheimer, B. R., \& Tinney, C. G. 2002a, MNRAS, 335, 1158

Clarke, F. J., Tinney, C. G., \& Covey, K. R. 2002b, MNRAS, 332, 361

Close, L. M., Siegler, N., Freed, M., \& Biller, B. 2003, ApJ, 587, 407

Dahn, C. C., Harris, H. C., Vrba, F. J., et al. 2002, AJ, 124, 1170

Donati, J.-F., Forveille, T., Cameron, A. C., et al. 2006, Sci, 311, 633

Dupuy, T. J., Liu, M. C., Bowler, B. P., et al. 2010, ApJ, 721, 1725

Enoch, M. L., Brown, M. E., \& Burgasser, A. J. 2003, AJ, 126, 1006

Forveille, T., Beuzit, J.-L., Delorme, P., et al. 2005, A\&A, 435, L5

Fuhrmeister, B., \& Schmitt, J. H. M. M. 2004, A\&A, 420, 1079

Gatewood, G., \& Coban, L. 2009, AJ, 137, 402

Gelino, C. R., Marley, M. S., Holtzman, J. A., Ackerman, A. S., \& Lodders, K. 2002, ApJ, 557, 433

Gizis, J. E., Monet, D. G., Reid, N. I., et al. 2000, AJ, 120, 1085

Goldman, B., Cushing, M. C., Marley, M. S., et al. 2008, A\&A, 487, 277

Güdel, M., \& Benz, A. O. 1993, ApJL, 405, L63

Hale, A. 1994, AJ, 107, 306

Hallinan, G., Antonova, A., Doyle, J. G., et al. 2006, ApJ, 653, 690

Hallinan, G., Antonova, A., Doyle, J. G., et al. 2008, ApJ, 684, 644

Hallinan, G., Bourke, S., Lane, C., et al. 2007, ApJL, 663, L25

Harding, L. K., Hallinan, G., Konopacky, Q. M., et al. 2013, A\&A, 554, A113

Jones, H. R. A., Pavlenko, Y., Viti, S., et al. 2005, MNRAS, 358, 105

Kirkpatrick, J. D., Henry, T. J., \& Irwin, M. J. 1997, AJ, 113, 1421

Koen, C. 2003, MNRAS, 346, 473

Koen, C. 2005, MNRAS, 357, 1151

Koen, C. 2006, MNRAS, 367, 1735

Koen, C. 2011, MNRAS, 411, 1197

Koen, C. 2012, MNRAS, 428, 2824
Koen, C., Matsunaga, N., \& Menzies, J. 2004, MNRAS, 354, 466 Konopacky, Q. M., Ghez, A. M., Barman, T. S., et al. 2010, ApJ, 711, 1087 Konopacky, Q. M., Ghez, A. M., Fabrycky, D. C., et al. 2012, ApJ, 750, 79

Lane, C., Hallinan, G., Zavala, R. T., et al. 2007, ApJL, 668, L163

Leggett, S. K., Allard, F., Geballe, T. R., Hauschildt, P. H., \& Schweitzer, A. 2001, ApJ, 548, 908

Liebert, J., Kirkpatrick, J. D., Cruz, K. L., et al. 2003, AJ, 125, 343

Littlefair, S. P., Dhillon, V. S., Marsh, T. R., et al. 2008, MNRAS, 391, L88

Lomb, N. R. 1976, Ap\&SS, 39, 447

Maiti, M. 2007, AJ, 133, 1633

Martín, E. L., Rebolo, R., \& Magazzu, A. 1994, ApJ, 436, 262

Martín, E. L., Zapatero Osorio, M. R., \& Lehto, H. J. 2001, ApJ, 557, 822

Mohanty, S., \& Basri, G. 2003, ApJ, 583, 451

Morin, J., Donati, J. F., Petit, P., et al. 2010, MNRAS, 407, 2269

Osten, R. A., Hawley, S. L., Bastian, T. S., \& Reid, I. N. 2006, ApJ, 637, 518

Osten, R. A., Phan-Bao, N., Hawley, S. L., et al. 2009, ApJ, 700, 1750

Phan-Bao, N., Osten, R. A., Lim, J., Martín, E. L., \& Ho, P. T. P. 2007, ApJ, 658,553

Radigan, J., Jayawardhana, R., Lafrenière, D., et al. 2012, ApJ, 750, 105

Reid, I. N., Burgasser, A. J., Cruz, K. L., et al. 2001, AJ, 121, 1710

Reid, I. N., Cruz, K. L., Laurie, S. P., et al. 2003, AJ, 125, 354

Reid, I. N., Kirkpatrick, J. D., Gizis, J. E., \& Liebert, J. 1999, ApJL, 527, L105

Reid, I. N., Kirkpatrick, J. D., Gizis, J. E., et al. 2000, AJ, 119, 369

Reid, I. N., Kirkpatrick, J. D., Liebert, J., et al. 2002, AJ, 124, 519

Reiners, A., \& Basri, G. 2007, ApJ, 656, 1121

Reiners, A., \& Basri, G. 2008, ApJ, 684, 1390

Reiners, A., \& Basri, G. 2009, ApJ, 705, 1416

Rockenfeller, B., Bailer-Jones, C. A. L., \& Mundt, R. 2006a, A\&A, 448, 1111

Rockenfeller, B., Bailer-Jones, C. A. L., Mundt, R., \& Ibrahimov, M. A. 2006b, MNRAS, 367, 407

Route, M., \& Wolszczan, A. 2012, ApJL, 747, L22

Rutledge, R. E., Basri, G., Martín, E. L., \& Bildsten, L. 2000, ApJL, 538, L141

Scargle, J. D. 1982, ApJ, 263, 835

Schwarzenberg-Czerny, A. 1991, MNRAS, 253, 198

Schweitzer, A., Gizis, J. E., Hauschildt, P. H., Allard, F., \& Reid, I. N. 2001, ApJ, 555,368

Sheehan, B., \& Butler, R. F. 2008, in AIP Conf. Proc. 984, High Time Resolution Astrophysics: The Universe at Sub-second Timescales (Melville, NY: AIP), 162

Stellingwerf, R. F. 1978, ApJ, 224, 953

Tinney, C. G. 1993, AJ, 105, 1045

Tinney, C. G., Reid, I. N., Gizis, J., \& Mould, J. R. 1995, AJ, 110, 3014

Tinney, C. G., \& Tolley, A. J. 1999, MNRAS, 304, 119

Vrba, F. J., Henden, A. A., Luginbuhl, C. B., et al. 2004, AJ, 127, 2948

West, A. A., \& Basri, G. 2009, ApJ, 693, 1289

West, A. A., Hawley, S. L., Walkowicz, L. M., et al. 2004, AJ, 128, 426

Zapatero Osorio, M. R., Martín, E. L., Bouy, H., et al. 2006, ApJ, 647, 1405 